\title{
Spectroscopy of baryon resonances
}

\author{
Reinhard Beck ${ }^{1, \star}$ and Ulrike Thoma ${ }^{1, \star \star}$ \\ ${ }^{1}$ Helmholtz-Institut für Strahlen- und Kernphysik, Universität Bonn, 53115 Bonn, Germany
}

\begin{abstract}
Within project A.1 of the SFB/TR16 "Subnuclear Structure of Matter", a large amount of data on photoproduction reactions has been accumulated at the Bonn Electron Stretcher Accelerator ELSA with the CBELSA/TAPS detector and was analysed in detail. In particular, data have been taken with unpolarized or with linearly or circularly polarized photons and with unpolarized or with longitudinally or transversely polarized protons. Photoproduction off neutrons was studied to determine the helicity amplitudes for the excitation of resonances off neutrons. In a partial wave analysis of the data, new resonances have been found and the properties of new and of known resonances have been determined, including the measurement of partial widths of so far unmeasured decay modes.
\end{abstract}

\section{Introduction}

The discovery of the $\Omega^{-}$baryon with strangeness $S=-3$ at the predicted mass in the reaction $K^{-} p \rightarrow\left(\Xi^{0} \pi^{-}\right) K^{+} K_{s}$ [1] established SU(3) symmetry and the conjecture that mesons and baryons are composed of constituent quarks $[2,3]$ and paved the path to an understanding of the particle zoo. Like the $\Delta^{++}(1232)$ and the $\Delta^{-}(1232)$, the $\Omega^{-}$ was interpreted as bound state containing three identical quarks (sss) while the two $\Delta(1232)$ were interpreted as $(u u u)$ and $(d d d)$ resonances. The spin and parity of these three baryons was $J^{P}=3 / 2^{+}$; with a quark spin $s=1 / 2$, the three quark spins had to be aligned to yield a total quark spin $S=3 / 2$. As ground states, these baryons had to have no intrinsic orbital angular momentum. However, with three identical quarks in a relative $S$-wave, this configuration was in conflict with the well-established Pauli principle which forbids two fermions with identical quantum numbers.

The solution was to introduce a further quantum number, color, and to postulate that three quarks with different colors (and a colored quark and an antiquark with anticolor) form a color singlet, and that the color wavefunction is antisymmetric with respect to the exchange of two colors $[4,5]$. The concept of color proved to be extremely important. From the request of local gauge invariance, a new theory of strong interactions, Quantum Chromo Dynamics or QCD, was deduced [6, 7] which assigned to quarks a new triple-valued charge called color. The fact that free quarks were never observed was understood by the hypothesis that color is confined [8], even though their mutual interactions were supposed to be weak at large momentum transfers or at small distances [9-11]. Even the baryon resonances seemed to be adequately de-

\footnotetext{
^e-mail: beck@hiskp.uni-bonn.de
}

${ }^{\star}$ e-mail: thoma@hiskp.uni-bonn.de scribed: Isgur and Karl demonstrated in their seminal papers that the hyperfine interaction between quarks suggested in QCD can, without further free parameters, explain the size and pattern of the splittings and the mixing angles observed experimentally in nonstrange, negative parity baryons [12]. The masses and mixing angles of the positive-parity baryons were also reasonably well reproduced in their QCD-inspired model [13]. In short: meson and baryon spectroscopy seemed to be well understood, and QCD was considered to be the established theory of strong interactions, with no mysteries and nothing more to learn. A survey of results on QCD from high-energy experiments can be found in an article by S. Bethke, G. Dissertori, and G. P. Salam [15] in the Review of Particle Properties RPP [16].

A few years before Isgur and Karl calculated the baryon spectrum, a family of new meson resonances had been discovered $[17,18]$ with the $\mathrm{J} / \psi$ as ground state. The interest of the particle physics community turned first to glueballs [19] and hybrids [20], then to the weakinteraction bosons [21, 22], to the Higgs particle [23], and to tests of the Standard Model (see, e.g. [24]).

It was realized only later that important physics was left behind unexplored. New theoretical concepts were formulated which needed to be confronted with experimental data but, on the experimental side, the evidence for a large number of nucleon and $\Delta$ resonances was challenged when new and precise data were included in the analysis. From a theoretical point of view, it was realized that low-energy approximations of QCD [25] lead to the Gell-Mann-Oakes-Renner relation [26] which assigns a mass of a few $\mathrm{MeV}$ to light (current) quarks while quark models assume a (constituent) quark mass of about $350 \mathrm{MeV}$. How is the mass generated? The mass gap between current and constituent quarks was interpreted by spontaneous breaking of the chiral symmetry expected for 
nearly massless quarks $[27,28]$. An important consequence is the large mass gap between chiral partners: the masses of the nucleon, with spin-parity $\mathrm{J}^{\mathrm{P}}=1 / 2^{+}$, and its chiral partner $N(1535) 1 / 2^{-}$, with spin-parity $\mathrm{J}^{\mathrm{P}}=1 / 2^{-}$ and mass $M=1535 \mathrm{MeV}$, differ by about $600 \mathrm{MeV}$. But it is not understood how QCD generates the structure of hadrons and how symmetries influence their structures and their dynamics.

An important development occurred in the realm of low-energy QCD [29]. QCD functions can be expanded in powers of the external momenta and of the quark masses. The Ward identities of chiral symmetry determine the expansion in terms of a few coupling constants (LECs) of a unique effective low-energy Lagrangian. Chiral Dynamics has become an rapidly expanding field (see [30] for a review), and is one of the cornerstones of the SFB/TR16 (see contributions of projects A.6, A.7, B.3, B.6, C.2, C.9 to these proceedings) and the $\mathrm{CRC} 110$ (projects A.2, A.3, A.4, A.5, B.6, B.7, B.8). Precise data on photoproduction of baryon resonances are an important ingredient for further advances of the field.

On the experimental side, in 1994 there were $17 \mathrm{~N}^{*}$ and $13 \Delta^{*}$ resonances below a mass of $2200 \mathrm{MeV}$ [31]. These resonances were all deduced from an analysis of data on $\pi N$ elastic and charge exchange scattering [32, 33]. These numbers are surprisingly low and, even worse, a large fraction of these resonances were not observed in the a later analysis based on a larger data sample [34] reporting $11 N^{*}$ and $9 \Delta^{*}$ only. The latter analysis included high precision data from the meson factories (see [34] for a list of data). This analysis predicted the spin rotation parameters [35-37] and the backward asymmetry [38] in the elastic pion-proton scattering from ITEP/PNPI very precisely while the predictions from $[32,33]$ show clear discrepancies with the data. Hence the number of trustworthy resonances was reduced to $11 N^{*}$ and $9 \Delta^{*}$ only.

At this point, we mention a basic problem in the analysis of data on $\pi N$ elastic and charge exchange scattering. The process is governed by two amplitudes. One of them represents a nucleon spin-flip in the scattering process, the other one a non-flip process. The two complex amplitudes are written in terms of two magnitudes and two phases. Two constraints come from experiment: the differential cross section $d \sigma / d \Omega$ and the asymmetry due to a target polarization $P$. Refs. [32, 33] make extensive use of dispersion relations to relate the ratio of the real to imaginary part of the scattering amplitude. In [34], an iterative procedure is used which includes energy-dependent fits to guide the solution. Thus there is an intrinsic under-determination of the scattering amplitude.

In the case of photoproduction, there are four complex amplitudes, nucleon spin-flip and non-flip, photon and nucleon spin aligned or anti-aligned. Thus at least seven (in fact eight) appropriate observables need to be determined in single pseudoscalar meson photoproduction, and one overall phase still remains open [60]. The measurement of a sufficient number of observables is the central theme of the efforts to measure the excitation spectrum of the nucleon in photoproduction. In general, if the tar- get and beam polarization can be controlled and the recoil polarization can be determined in an experiment, even 16 observables become accessible overdetermining the amplitudes.

At a first glance it seems that $\pi N$ elastic and charge exchange scattering or pion-induced inelastic reactions might be the better choice to study the nucleon excitation spectrum since fewer observables need to be measured. However, the larger number of contributing amplitudes results in a larger number of interfering amplitudes, and the interference between large and small amplitudes increases the sensitivity to tiny effects. This is a great advantage of photoproduction experiments. A second advantage is that resonances with very small coupling to $\pi N$ but "normal" $\gamma N$ couplings may become experimentally accessible. The de-coupling of resonances from $\pi N$ was made responsible for the non-observation of the many resonances predicted by quark models.

\section{What has been reached?}

Before discussing in detail the outcome of the experiments carried out within the A.1 project of the SFB/TR16, we would like to mention here the main achievements. In 2011, we performed a first systematic search for new baryon resonances [39-42]. A very important result was that the resonances proposed by the groups led by Höhler [32] and Cutkosky [33] were confirmed in the BonnGatchina $(\mathrm{BnGa})$ partial wave analysis (see project A.2 "Partial Wave Analysis"). This analysis included $\pi N$ scattering data but the results were largely based on the new photoproduction data taken within the SFB/TR16 and at other places. Thirteen resonances were observed which were not seen in the most recent analysis based on $\pi N$ elastic scattering data [34]. There were six new entries in the

Table 1. New resonances found in the BnGa-PWA, resonances for which the star rating was improved, and resonances confirmed by the BnGa-PWA but not seen in the GWU-Arndt'06-analysis.

\begin{tabular}{|l|c|c|c|c|}
\hline & RPP & our & RPP & GWU'06 \\
analyses & 2012 & \\
\hline $\mathrm{N}(1700) 3 / 2^{-}$ & $* * *$ & $* * *$ & $* * *$ & no evidence \\
$\mathrm{N}(1710) 1 / 2^{+}$ & $* * *$ & $* * *$ & $* * *$ & no evidence \\
$\mathrm{N}(1860) 5 / 2^{+}$ & & $*$ & $* *$ & \\
$\mathrm{~N}(1875) 3 / 2^{-}$ & & $* * *$ & $* * *$ & no evidence \\
$\mathrm{N}(1880) 1 / 2^{+}$ & & $* *$ & $* *$ & no evidence \\
$\mathrm{N}(1895) 1 / 2^{-}$ & & $* *$ & $* *$ & no evidence \\
$\mathrm{N}(1900) 3 / 2^{+}$ & $* *$ & $* * *$ & $* * *$ & no evidence \\
$\mathrm{N}(1990) 7 / 2^{+}$ & $* *$ & $* *$ & $* *$ & no evidence \\
$\mathrm{N}(2000) 5 / 2^{+}$ & $* *$ & $* *$ & $* *$ & no evidence \\
$\mathrm{N}(2060) 5 / 2^{-}$ & & $* * *$ & $* *$ & no evidence \\
$\mathrm{N}(2150) 3 / 2^{-}$ & & $* *$ & $* *$ & no evidence \\
$\Delta(1900) 1 / 2^{-}$ & $*$ & $*$ & $* *$ & no evidence \\
$\Delta(1920) 3 / 2^{+}$ & $* * *$ & $* * *$ & $* * *$ & no evidence \\
$\Delta(1940) 3 / 2^{-}$ & $*$ & $* *$ & $* *$ & no evidence \\
\hline
\end{tabular}


Table 2. Properties of the $N(1900) 3 / 2^{+}$resonance from the BnGa multichannel partial wave analysis. Along with the name of the resonance, the star rating of the Particle Data Group [16] is given. BnGa suggested an upgrade to four stars, marked by $\mathrm{a} \star$. The helicity couplings $A_{1 / 2,3 / 2}$ are given in $\mathrm{GeV}^{-\frac{1}{2}}$. The electromagnetic transition amplitudes $\gamma p, \gamma p^{1 / 2}, \gamma p^{3 / 2}$ are defined as elements of the transition matrix and dimensionless. $\pi N \rightarrow \pi N$ stands for the elastic pole residue, $2(\pi N \rightarrow X) / \Gamma$ for inelastic pole residues. They are normalized by a factor $2 / \Gamma$ with $\Gamma=\Gamma_{\text {pole }}$.

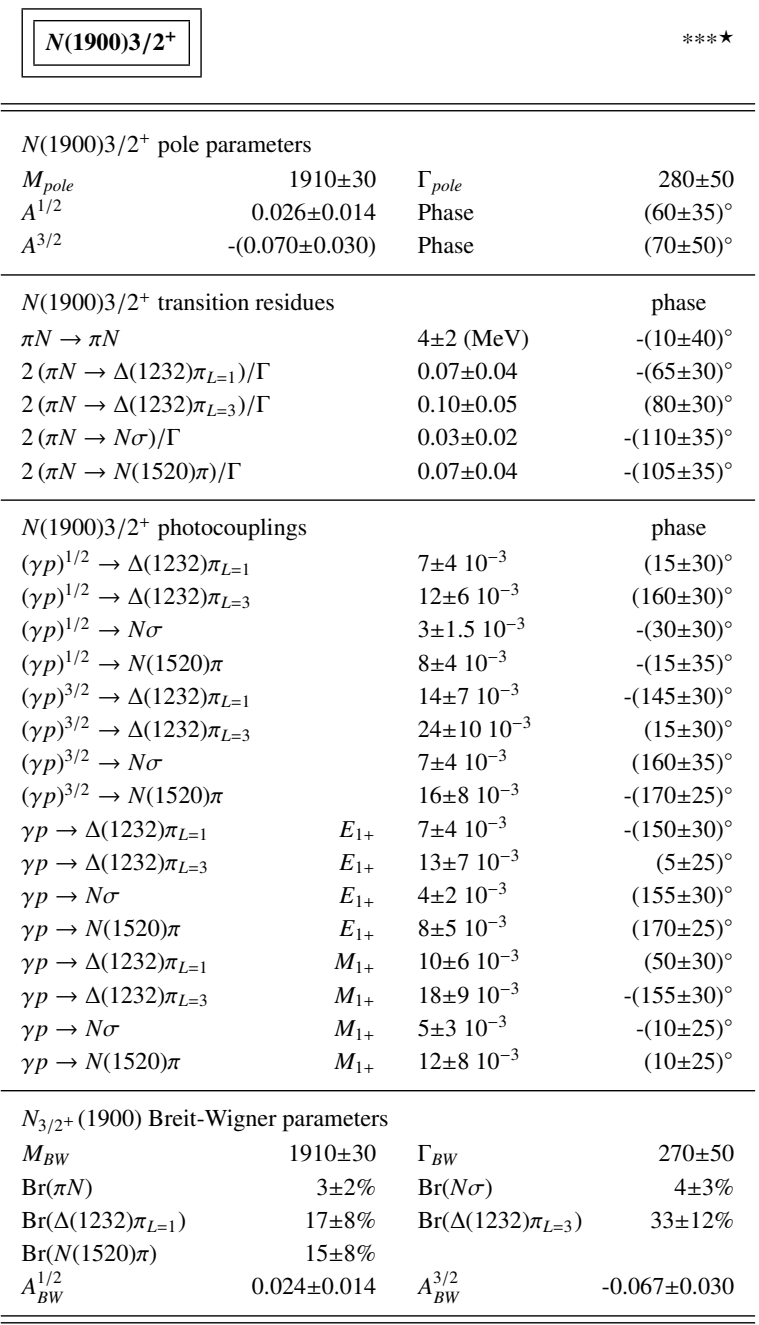

Review of Particle Properties, and two resonances were upgraded in their star-rating because of the BnGa analysis. This success underlines the impact photoproduction experiments can make in the study of baryon resonances. Table 1 lists the impact of the new photoproduction data on the number of observed resonances. At present, we perform a new systematic search for missing resonances based on the data taken up to now. The fits are performed with five further resonances not yet included in the Review of Particle Properties. It is unclear at present, how significant these are, if some resonances will be upgraded due to the new study, and whether further resonances will be required to achieve a good fit.

A further important step forward are new informations about the properties of baryon resonances. In the recent years, we included the reactions $\gamma p \rightarrow p \pi^{0} \eta[43-48]$ and $\gamma p \rightarrow p \pi^{0} \pi^{0}$ [49-57] into the BnGa data base. As a result, more than 500 resonance properties derived from these analyses are listed in the 2016 edition of the Review of Particle Properties [58]. Table 2 gives the properties of $N(1900) 3 / 2^{+}$as an example. The $\pi N$ branching ratio is rather small: this is the reason why it was not seen in [3234]. The sum of the observed branching ratios as given in table 2 is $72 \pm 14 \%$. Measured are also branching ratios for decays into $N \eta(10 \pm 4 \%), \Lambda K(13 \pm 5 \%)$, and $\Sigma K(6 \pm 3 \%$ [39]), and into $N \omega(15 \pm 8 \%$ [59]). The total sum is already more than, but compatible with, $100 \%$, leaving little room for decays into $N \rho$. These are presently under study. This example shows the strength of a coupled channel analysis: the sum of all decay branching ratios of a trustworthy resonance must be compatible with or at least close to 1 .

Not all the quantities listed in Table 2 are physically significant. Nevertheless they demonstrate that our knowledge on the spectrum of nucleon excitations has increased very substantially.

\section{The CBELSA/TAPS experiment at ELSA}

\subsection{Observables}

Fig. 1 shows the total cross section for $\gamma p \rightarrow p \pi^{0}$, together with the Breit-Wigner shapes of the contributing resonances based on RPP values for masses, widths, $N \pi$ decay branching ratios and helicity amplitudes. There are clear peaks which can be assigned to photoproduction of $\Delta(1232) 3 / 2^{+}, N(1520) 3 / 2^{-}$, and $N(1680) 5 / 2^{+}$. But there are more resonances which are not seen as obvious peaks: baryon resonances are broad and overlap and, obviously, small resonance contributions are difficult to extract from the data. The resonances appear in different partial waves, and these need to be separated and identified. As mentioned in the introduction, photoproduction is governed by

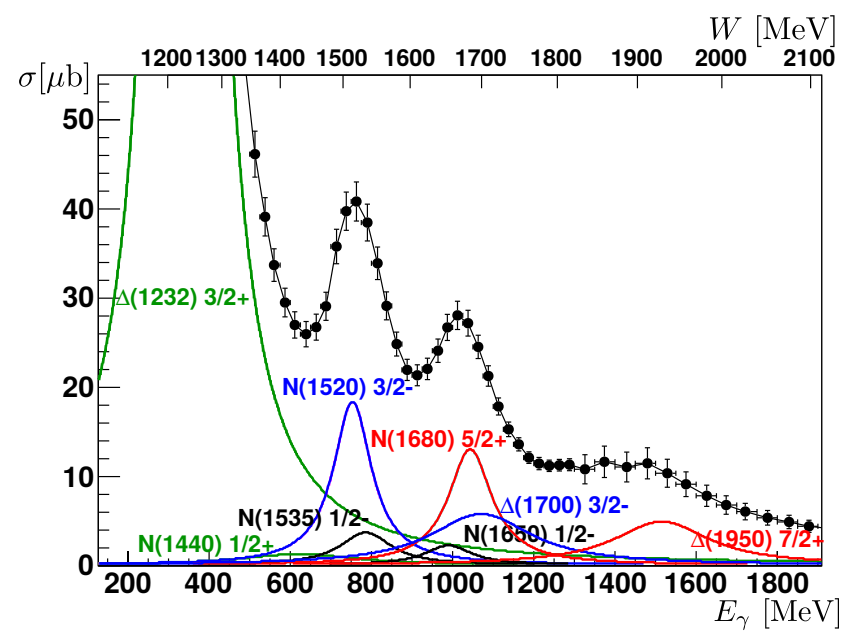

Figure 1. $\gamma p \rightarrow p \pi^{0}$ total cross section (CBELSA/TAPS-data), also indicated are the pure Breit-Wigner shapes of the different resonances contributing to the reaction. The phase space dependence is not included here and the couplings are taken from the RPP [16]. $\pi N-\mathrm{P}-,-\mathrm{D}-$, and -F-wave resonances are shown in green, blue and red, respectively. 
Table 3. Observables in single pseudoscalar meson photoproduction. Measurements of the observables marked in blue have been performed within the SFB/TR16 for various final states with neutral mesons.

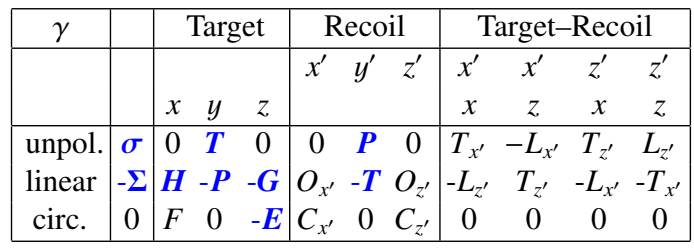

four complex amplitudes; for an energy-independent reconstruction of the amplitudes, at least eight carefully chosen observables need to be measured [60] even though still one phase per interval in energy and angle remains undetermined.

Alternatively, energy-dependent fits can be made like Bonn-Gatchina (A.2), Jülich-Bonn (B.3), or Gießen (B.7), or multipole analyses in which the angular dependence is exploited in the first step. Truncated multipole analyses require measurements of a smaller number of observables [61]; a first attempt in [62] was successful.

Table 3 shows the 16 observables which are experimentally measurable. These polarization observables, or at least 8 carefully chosen ones [60], need to be measured over the full solid angle with high precision. This aim defines the requirements the photon beam, the target and the detector to study light-baryon resonances should meet: The electron accelerator needs to deliver unpolarized or polarized electrons with a large duty cycle from which an intense well collimated photon beam with no, linear, or circular polarization can be produced. Of course the energy of the photon beam should span an energy range which allows to cover the excitation spectrum. A target, with protons or "neutrons", should be polarizable in longitudinal (along the photon beam) or transverse direction. No polarization is required to measure the differential cross section $\mathrm{d} \sigma / \mathrm{d} \Omega$. The beam asymmetry $\Sigma$ is given by the difference of two perpendicular settings of the linear photon polarization plane. When the target is polarized perpendicular to the beam direction, $T$ can be determined. If we do not consider the difficult measurement of the recoil polarization, all other observables require then both, photon and target to be polarized. An ideal detector would measure charged and neutral (i.e. photons) particles with high precision and complete solid angle coverage.

For two-meson production or the production of mesons with non-zero spin, the number of observables to be measured is significantly higher. Additional observables become accessible using a polarized beam and/or target. Following [63] at least 15 carefully chosen observables need to be measured for double pseudoscalar meson photoproduction. Additional observables can be deduced with the same kind of polarization settings as in the case of photoproduction of one pseudoscalar meson. However, to achieve a complete experiment in double-pseudoscalar meson photoproduction also triple-polarization observables need to be measured. The latter are not needed in the case of single-pseudoscaler meson photoproduction.
In Bonn, the ELectron Stretcher Accelerator ELSA [64] with its possibility to accelerate polarized electrons [66] (see contribution of project D.2 to these proceedings) and its goniometry to produce linear photon polarization [67] fulfills the requirements discussed. Unpolarized as well as circularly and linearly polarized photon beams are available for the experiments. In addition, the Bonn frozen spin polarized target [68] makes polarized protons and "neutrons" available (see contribution of project D. 1 to these proceedings). Using the CBELSA/TAPS experiment, the polarization observables marked in blue in Table 3 have been determined within project A.1 for various different final states. Depending on their cross sections, additional statistics is often still desirable.

\subsection{Detector requirements}

The missing resonances were expected to decouple from the $\pi N$-channel [69]. Obviously, it is difficult or even impossible to observe baryon resonances with very small or nearly vanishing $\pi N$ coupling in $\pi N$ elastic and charge exchange scattering, or in photoproduction of single pions. However, baryon resonances have to decay and may couple with rather different strengths to different final states. The study of various final states, including the multimeson photoproduction, is necessary to gain a complete picture of the spectrum and the properties of nucleon excitations.

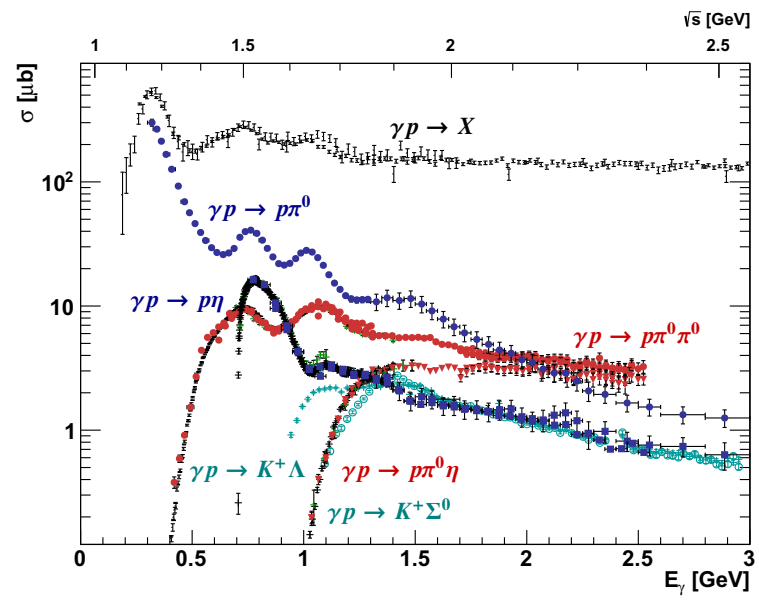

Figure 2. Total photon absorption cross section as well as total cross sections for different final states: $\gamma p \rightarrow X$ [58], $\gamma p \rightarrow \pi^{0} p$ [70-72], $\gamma p \rightarrow p \eta[73-79], \gamma p \rightarrow K^{+} \Lambda[80,81], \gamma p \rightarrow K^{+} \Sigma^{0}[80,81], \gamma p \rightarrow$ $p \pi^{0} \eta[45,46,48], \gamma p \rightarrow p \pi^{0} \pi^{0}[49-54,57]$

Figure 2 shows the inclusive total cross section for $\gamma p \rightarrow X$ [58], and the contributions from different final states. At low energies, the formation of $\Delta(1232) 3 / 2^{+}$ dominates photoproduction. Over a wide range, the inclusive total cross section adopts a value of about $200 \mu \mathrm{b}$. The $\gamma p \rightarrow p \pi^{0}$ cross section falls off rapidly with energy; the peaks have been assigned to $\Delta(1232) 3 / 2^{+}$, and dominantly to $N(1520) 3 / 2^{-}$, and $N(1680) 5 / 2^{+}$(of course also other resonances with smaller cross section contribute to the second and third resonance region, see also Fig. 1). The 


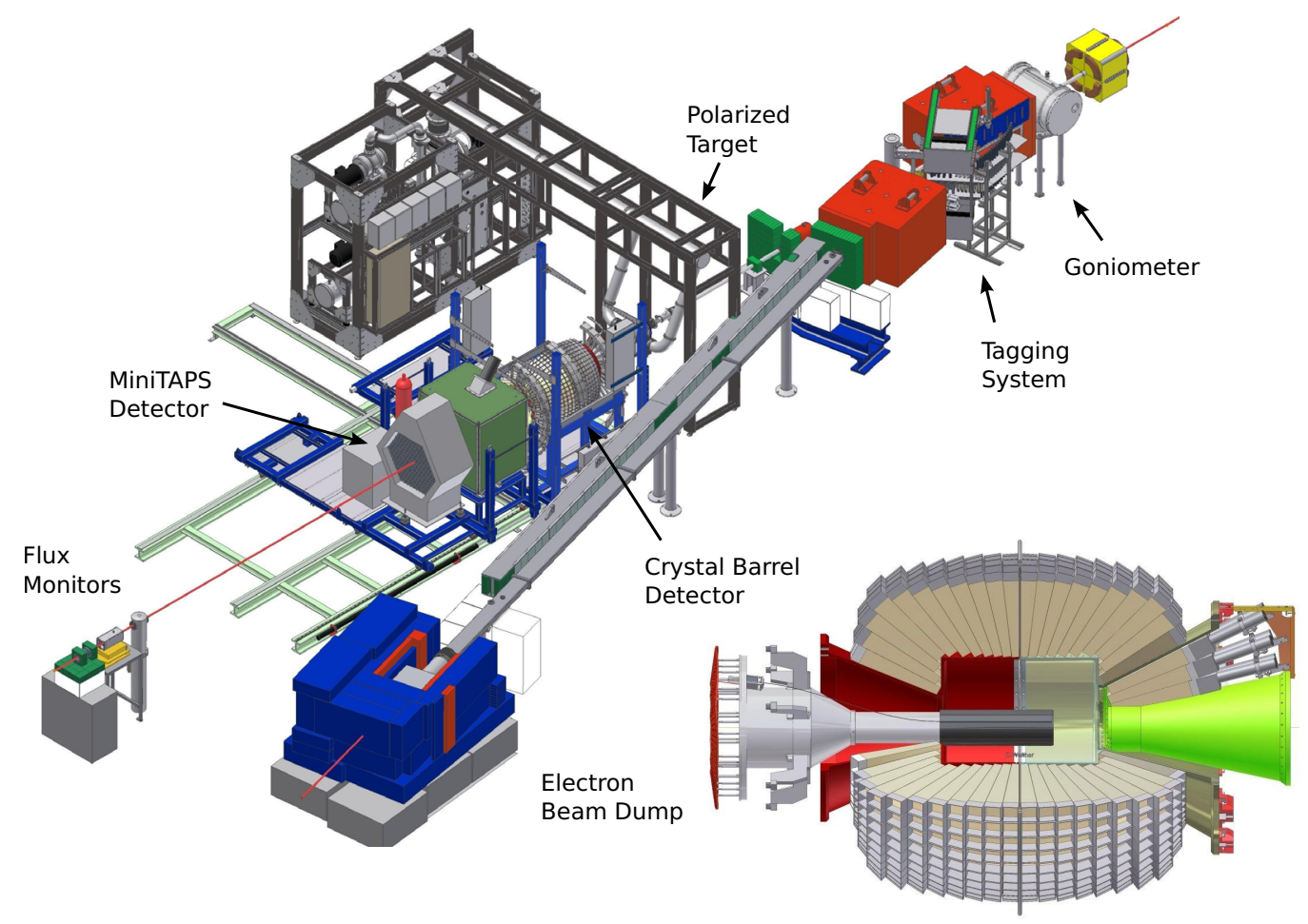

Figure 3. Experimental Setup of the CBELSA/TAPS Experiment.

small additional enhancement at $E_{\gamma}=1.5 \mathrm{GeV}$ is mostly due to $\Delta(1950) 7 / 2^{+}$. Above the threshold of the reaction, the $\gamma p \rightarrow p \eta$ cross section is dominated by formation of $N(1535) 1 / 2^{-}$. The two reactions with open strangeness in the final state shown $\left(\gamma p \rightarrow K^{+} \Lambda\right.$ and $\left.\gamma p \rightarrow K^{+} \Sigma\right)$ have cross sections which are, at intermediate energies, in size similar to the $\gamma p \rightarrow p \eta$ cross section. The reactions $\gamma p \rightarrow \pi^{0} \pi^{0} p$ and $\gamma p \rightarrow \pi^{0} \eta p$ gain importance with increasing energy. A comparison of the different total cross sections by eye already indicates that different resonances couple with rather different strengths to the different final states. This clearly indicates the importance of the analysis of different final states to gain a complete understanding of the spectrum. The large gap between the inclusive total cross sections and the cross sections for exclusive final states is mainly due to reactions like $\gamma p \rightarrow \pi^{+} \pi^{-} p, \pi^{+} \pi^{0} n$, and $3 \pi N$.

The ideal detector would hence measure the momenta of charged and neutral final-state particles and identify photons and neutrons, and charged pions, kaons, and protons. In practice, present-day detectors are either very well suited for the measurements of charged or neutral decay products. The CBELSA/TAPS detector (section 3.3) is ideally suited to measure neutral mesons decaying into photons. This is advantageous since background amplitudes like charged pion t-channel exchange or KrollRudermann-terms do not contribute. Hence the fractional contribution of the resonant amplitudes is larger than for reactions with charged mesons in the final state. Project B.1 of this SFB added further final states such as e.g. $K^{0} \Sigma^{+}$[84]. The BGO-OD-experiment provides the option to measure charged particles in forward direction with very good resolution. For further details on the BGOOD-experiment and its capabilities to measure charged and neutral particles, see the contribution of project B.1 to these proceedings.

The CLAS detector at JLab is very well suited to measure charged particles, making data on additional final states such as e.g. $K^{+} \Lambda$ and $K^{+} \Sigma^{0}$ or $p \pi^{+} \pi^{-}$photoproduction available [82, 83]. For $K^{+} \Lambda$ and $K^{+} \Sigma^{0}$ photoproduction, CLAS (Jlab) has e.g. performed not only all measurements needed to reach a complete experiment, but all 16 polarization observables have been measured. In this case the self-analyzing power of the hyperon-decay gives directly access to the polarization of the baryon in the final state, which is of course of great advantage.

A combined analysis of these data sets in a multichannel PWA (see project A.2 "Partial Wave Analysis") is of great advantage compared to the analysis of individual channels to extract the resonances from the data. All data constrain the solution substantially. A similar argument holds for the photoproduction off the proton and neutron. While the isospin-3/2- $\Delta$-resonances are produced with the same strength off the proton and the neutron, this is not true for the isospin-1/2- $N^{*}$-resonances. Some $N^{*}$ resonances might be strongly produced off the neutron but not off the proton or vice versa. Therefore resonances, maybe even resonances with specific symmetries in their wave function or nature, may escape detection if only proton data are analyzed. While first neutron data have been taken within this SFB, neutron data are still scarce. A combined PWA of proton and neutron data will further constrain the different isospin contributions. 


\subsection{The CBELSA/TAPS experiment at ELSA}

Fig. 3 shows an overview of the CBELSA/TAPS experiment. The experiment is located at the electron accelerator ELSA [64]. ELSA provides either unpolarized or longitudinally polarized electrons with energies up to $3.5 \mathrm{GeV}$ (for further details see contribution of project D. 2 to these proceedings). The electrons hit a radiator target placed inside of a goniometer. Here photons are produced via bremsstrahlung. The electrons which underwent the bremsstrahlung process are momentum analyzed in the tagging system. They are deflected in a magnetic field and detected in a ladder of organic scintillator bars and fibres. The bremsstrahlung photons hit an unpolarized or polarized target (see contribution of project D. 1 "Polarized Target" to these proceedings), which is surrounded by a three-layer scintillation fiber detector (513 fibers) used for the identification of charged particles and by an electromagnetic calorimeter consisting of $1230 \mathrm{CsI}(\mathrm{Tl})$-crystals (part of the former Crystal Barrel calorimeter used at the Low Energy Antiprotion Ring LEAR at CERN [85]). In the forward direction below polar angles of $30^{\circ}$, two further calorimeters, the Forward Plug (90 CsI(Tl)-crystals) and the forward TAPS-wall $\left(216 \mathrm{BaF}_{2}\right.$ crystals) are placed. Plastic scintillators in front of the crystals allow charged particle identification. Inbetween the Crystal Barrel and the TAPS detector, a $\mathrm{CO}_{2}$ Cherenkov detector is placed to suppress electromagnetic background produced in the target.

For the data discussed within these proceedings the signals from the forward calorimeters, the fiber detector and the tagging system were used as first-level trigger. The Crystal Barrel calorimeter was only included in the second-level trigger identifying the number of clusters in the calorimeter. Presently the calorimeter readout is upgraded to provide in future also timing and first level trigger capabilities of the calorimeter as discussed in detail in the contribution of project D. 3 to these proceedings.

\subsection{Polarized photon beams}

The bremsstrahlungs process produces an unpolarized photon beam with approximately a $1 / E_{\gamma}$ intensity distribution. Linear photon polarization is obtained when electrons are scattered off a diamond radiator. Different settings of the diamond radiator are required to maximize the polarization in the desired energy region (see Fig. 4).

The bremstrahlungs-photons are polarized circularly when they are produced by longitudinally polarized electrons. For data with circularly polarized photons, a Møller target is used as bremsstrahlung target to monitor the electron polarization via Møller scattering in parallel to the data taking. The electron polarization was typically $64 \%$; the polarization transfer decreases with decreasing photon energy according to [65]:

$$
P_{\odot}=\frac{4 x-x^{2}}{4-4 x+3 x^{2}} \quad \text { with } x=\frac{E_{\gamma}}{E_{\mathrm{el}}} .
$$

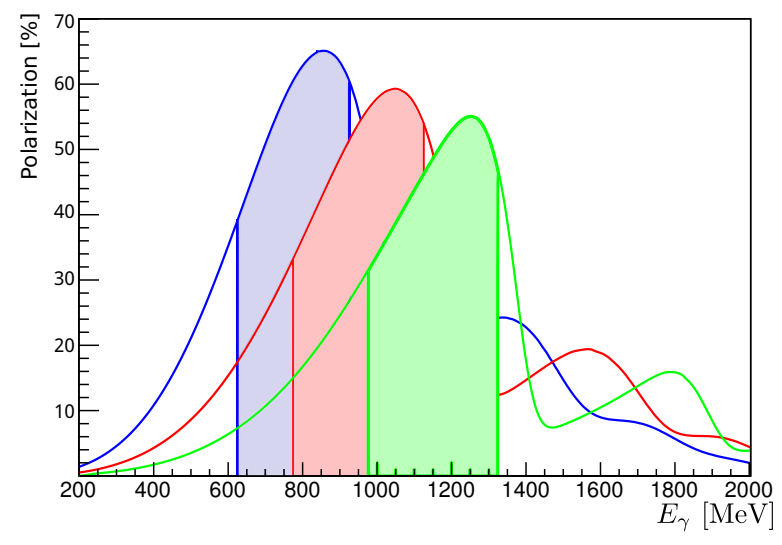

Figure 4. Three different settings of the coherent edge in the 2008 data taking period, the shaded areas mark the energy regions used in this analysis (from [86, 87]).

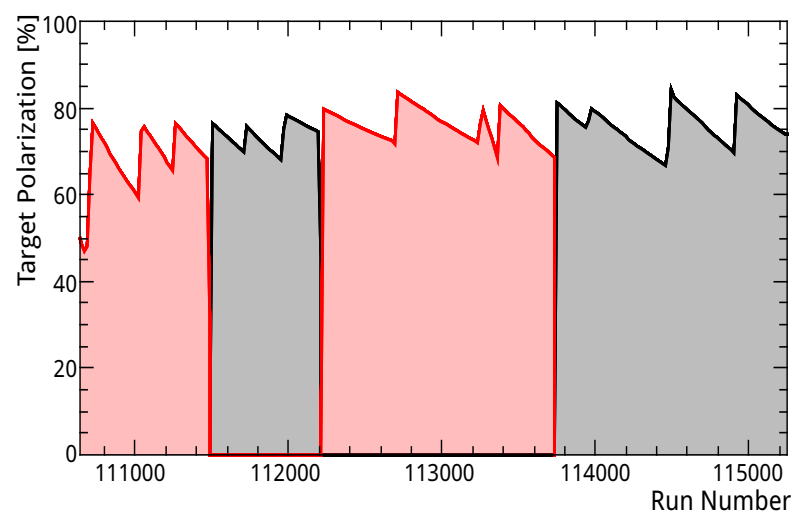

Figure 5. The target polarization during the 2008 beamtime of 40 days with positive (red) and negative (black) polarization (from [87]).

\subsection{The polarized target}

Double polarization experiments have been performed using the Bonn polarized frozen spin butanol target (project D.1) reaching typical mean polarizations of about $70-75 \%$ during the measurements.

Since the target $\left(\mathrm{C}_{4} \mathrm{H}_{9} \mathrm{OH}\right)$ consists not only of hydrogen but is diluted by the carbon and oxygen, measurements using a carbon foam target have been performed to determine the so-called dilution factor $(d)$. The carbon foam target was included in the cryostat as otherwise the butanol. To reach similar conditions the target had approximately the same target area density and dimensions as the unpolarizable components in the butanol target. Measurements using a liquid hydrogen target were used for further systematic studies. The dilution factor depends on the kinematic variables (e.g. $E_{\gamma}, \theta$ for two-particle final states). One example for an analysis using the $\mathrm{C}$ foam-target to determine the dilution factor is shown below (Fig. 6). A corresponding dilution factor

$$
d\left(E_{\gamma}, \theta\right)=\frac{N_{\text {butanol }}-N_{\text {carbon }}}{N_{\text {butanol }}}
$$


needs to be determined for each specific analysis. The obtained values of course depend on the cuts. The more restrictive the cuts are, the higher becomes the dilution factor. For forward going pions a smaller fraction of protons is detected in the calorimeter. The cuts are less restrictive, therefore more events stemming from the bound nucleons remain and the dilution factor decreases (Fig. 6).

\section{$4 \pi^{0}, \eta$, and $\eta^{\prime}$ photoproduction}

\subsection{Differential cross sections and the beam asymmetry $\Sigma$}

Differential cross sections for the reactions $\gamma p \rightarrow \pi^{0} p$ $[70,71]$ and $\gamma p \rightarrow \eta p[73,74]$ have been measured at the beginning of the SFB, followed by additional measurements of $\gamma p \rightarrow \pi^{0} p$ [72] and $\gamma p \rightarrow \eta p$ and $\gamma p \rightarrow \eta^{\prime} p$ [77] covering the full solid angle also in forward direction. Recently, differential cross sections and spin density matrix elements were determined for single $\omega$ meson photoproduction off the proton [88]. The reaction $\gamma p \rightarrow \pi^{0} \omega p$ was shown to be dominated by $\Delta(1232) \omega$ production [89]. Measurements of the beam asymmetry were carried out for various final states: $p \pi^{0}[90,91], p \eta$ [67], $p \omega$ [92], and $K_{s}^{0} \Sigma^{+}$[84], (see also contribution of project B.1 to these proceedings).

\section{$4.2 \gamma p \rightarrow \pi^{0} p$ : The double polarization observable $G$}

Within the SFB measurements with a linearly polarized photon beam and a longitudinally polarized target have been performed. The linearly polarized photons stem from coherent bremstrahlung of electrons with an energy of
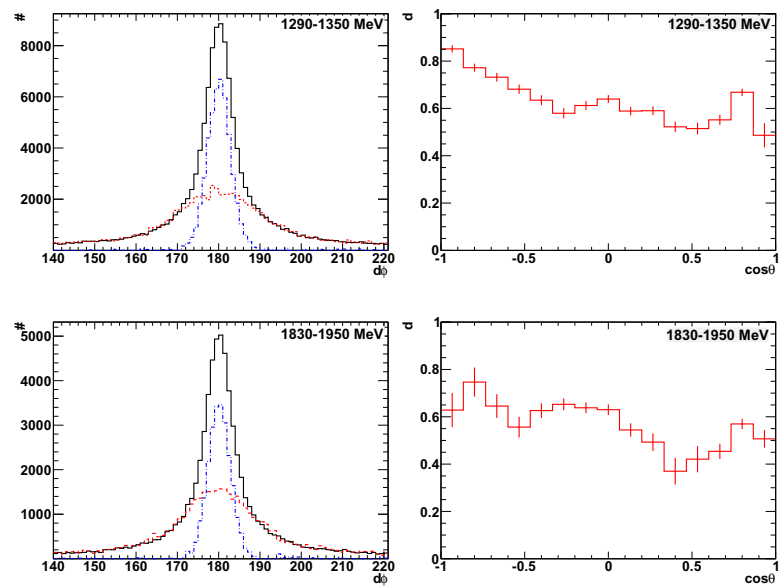

Figure 6. Determination of the dilution factor; as an example the analysis of $\gamma p \rightarrow p \pi^{0}$ using a longitudinally polarized target and a circularly polarized beam was chosen. Left: Coplanarity distribution used to determine the fraction of events in the butanol data produced off bound nucleons in carbon (oxygen), black: butanol data, red: carbon data, blue: resulting hydrogen spectrum. The coplanarity spectrum of the carbon data was adjusted to the butanol data outside the central peak area where the free protons contribute. The resulting scaling factor is then used to determine the dilution factor (right) depending on the angle [101].

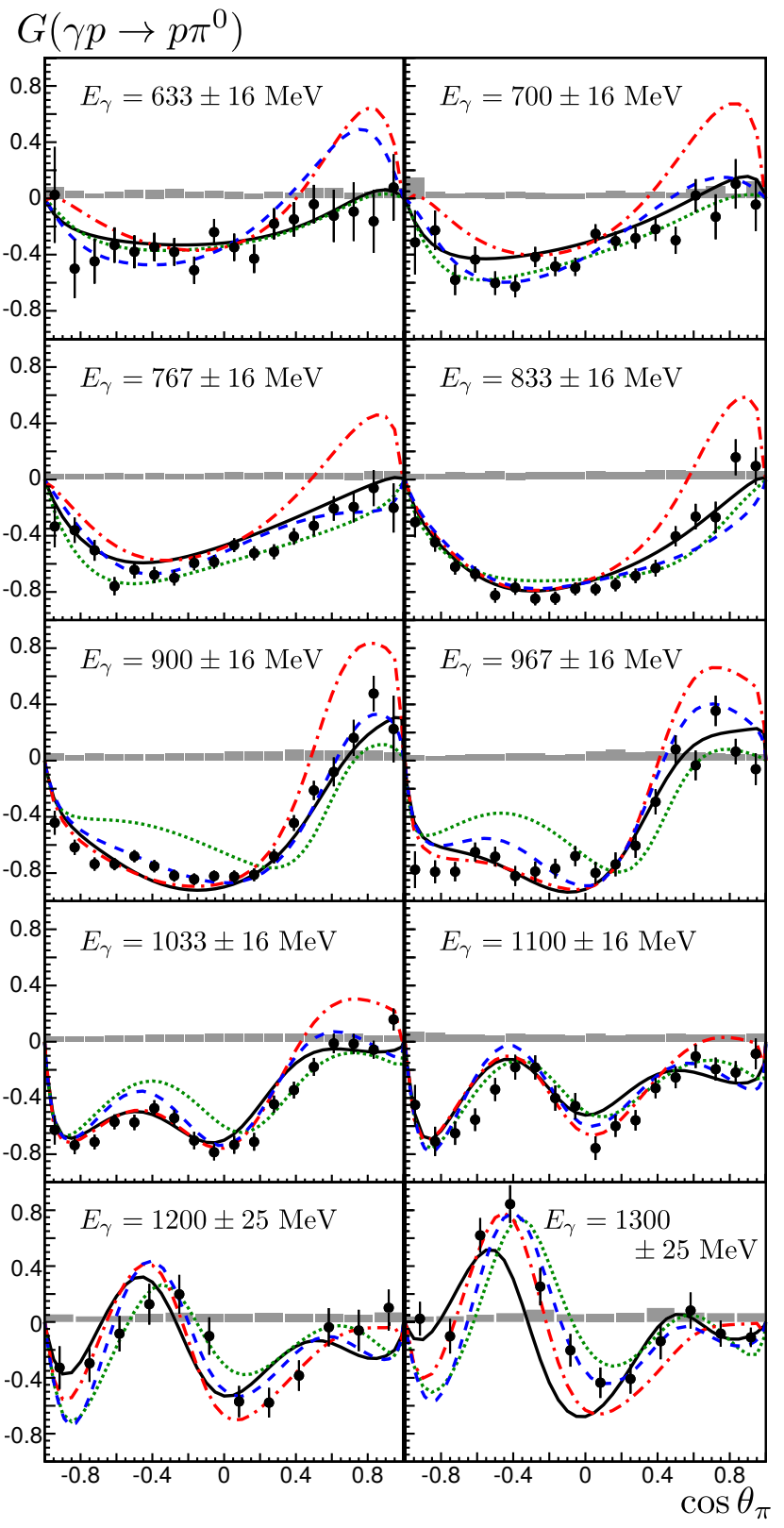

Figure 7. The double-polarization observable $G$ for every second measured photon energy bin [87] (black dots), compared to the PWA predictions: MAID 2007 [93] (green dotted line), SAID CM12 [94] (red dashed-dotted line), JüBo 2013-01 [95] (blue dashed line), and BnGa 2011-02 [42] (black solid line).

3.2 $\mathrm{GeV}$ off an aligned diamond. The corresponding cross section can be written as:

$$
\frac{\mathrm{d} \sigma}{\mathrm{d} \Omega}(\phi)=\frac{\mathrm{d} \sigma_{0}}{\mathrm{~d} \Omega} \cdot\left(1-p_{\gamma}^{\operatorname{lin}} \Sigma \cos (2 \phi)+P_{\gamma}^{\mathrm{lin}} p_{z} G \sin (2 \phi)\right)
$$

where $\frac{\mathrm{d} \sigma_{0}}{\mathrm{~d} \Omega}$ is the unpolarized cross section, $p_{\gamma}^{\text {lin }}$ the degree of linear polarization, $p_{z}$ the polarization of the target and $\phi$ the angle between the polarization and the production plane. Data have been taken with three settings for the linear polarization with coherent edges at $950 \mathrm{MeV}$, $1150 \mathrm{MeV}, 1350 \mathrm{MeV}$, the maximum linear polarization reached was $65 \%$ at $860 \mathrm{MeV}, 59 \%$ at $1050 \mathrm{MeV}$, and $55 \%$ at $1270 \mathrm{MeV}$ (see Fig. 4). 
The final results for our new $G$ data [87] are presented in Fig. 7. It shows the double-polarization observable $G$ as a function of $\cos \theta_{\pi}$ for photon energies from $E_{\gamma}=617 \mathrm{MeV}$ to $E_{\gamma}=1325 \mathrm{MeV}$. For every second energy bin, the data are shown in black with their statistical errors; the systematic errors are given as gray histogram on the 0 -axis. Symmetry principles enforce $G=0$ for $\cos \theta_{\pi}= \pm 1$. Data at exactly these points do not exist but the most forward or backward data points are compatible with a PWA-curve vanishing at $\cos \theta_{\pi}= \pm 1$. At low photon energies, for $E_{\gamma}<900 \mathrm{MeV}$, the values of $G$ as functions of $\cos \theta_{\pi}$ all show negative values, with a single minimum at negative values of $\cos \theta$. With increasing photon energy, the distributions become more complicated, with up to three local minima and two local maxima.

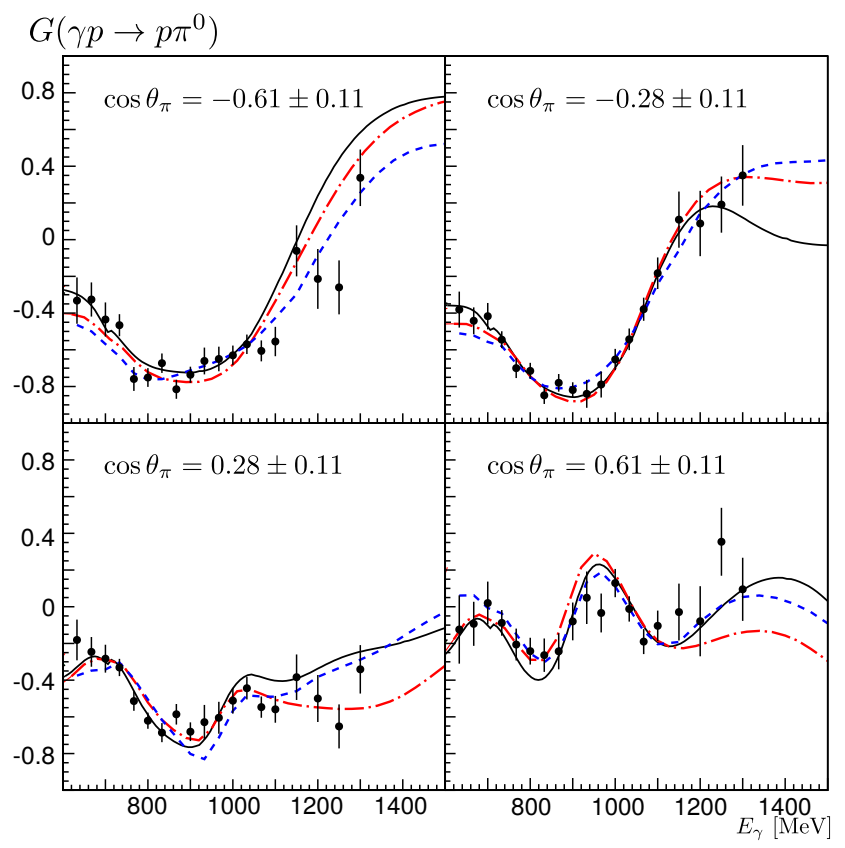

Figure 8. The energy dependence of $G$ [87] for four different angles, compared to different solutions of the PWAs: BnGa 201402 [48] (black solid line), SAID [97, 99] (red dashed-dotted line), and JüBo $[98,99]$ (blue dashed line).

The new data for $\mathrm{G}$ are compared in Fig. 7 with predictions from different PWA models: with BnGa 2011-02 [42], MAID 2007 [93], SAID CM12 [94], and JüBo 201301 [95]. The comparison reveals that at lower energies, the MAID and BnGa analyses can describe the data well, while for the SAID results, a deviation at $\cos \theta_{\pi} \geq 0.4$ becomes apparent. The predictions of JüBo show a similar disagreement as SAID for the two lowest photon energy bins at $E_{\gamma}=633$ and $667 \mathrm{MeV}$. The differences between the predictions of the MAID model and the data in the medium energy region were discussed in [86] and traced back to the multipoles $E_{0+}$ and $E_{2-}$. The largest deviations between the different models can be observed in the higher energy bins $\left(E_{\gamma}>1150 \mathrm{MeV}\right)$. These differences most likely occur since resonance contributions in the fourth resonance region are not well known.
The new $G$ data were included in the BnGa, JüBo, and the SAID analyses and new fits were performed. The new fits, BnGa 2014-02 [48] (black solid line), SAID [97] (red dashed-dotted line), and JüBo [98] (blue dashed line), reproduce the data reasonably well [87]. For convenience, we show the data in Fig. 8 for four slices in $\cos \theta$ as a function of the photon energy. All PWA fits can describe the new data very well at lower photon energies, above $E_{\gamma}>1150 \mathrm{MeV}$ the fit results start to diverge. Here more precise data for $G$ are needed to constrain the PWA solutions.

The impact of the new data can be best seen when the multipoles $E_{0+}, E_{2-}$ and $M_{2-}$ exciting the dominantly contributing resonances are compared; a longer paper with a comparison of all leading multipoles has been published recently [99]. The values of the double-polarization observable $G$ reported in this subsection have been used together with the data on the observables $E[100,101]$, and $T, P$, and $H[102,103]$. As an example, the real and imaginary parts of the $E_{0+}$ multipole derived from these new fits to the data are shown in Fig. 9 and compared with the $E_{0+}$ multipole derived from the older fits. For the imaginary part of the multipole, the spread of the solutions reduces considerably: the new data with double-polarization observables $(G, E, T, P, H)$ have a decisive influence on the resulting multipoles. For the real part, some reduction in the spread is observed even though much less pronounced. Certainly, more precise polarization data and further analyses are both required before the remaining discrepancies are resolved.

These new data had a decisive impact on the $\pi$-photoproduction multipoles. When these data and other new data were included in the partial-wave analyses BonnGatchina and SAID and in the dynamical coupled-channel approach Jülich-Bonn, the mutual agreement was improved considerably [99].

\section{$4.3 \gamma p \rightarrow \pi^{0} p:$ The double polarization observable $E$}

The helicity asymmetry $E$ requires circularly polarized photons and longitudinally polarized protons. It can be determined as

$$
E=\frac{N_{1 / 2}-N_{3 / 2}}{N_{1 / 2}+N_{3 / 2}} \cdot \frac{1}{d} \cdot \frac{1}{p_{\odot} p_{T}}
$$

where $N_{1 / 2}$ and $N_{3 / 2}$ are the number of events observed with photon and target polarization in opposite or parallel directions, normalized to the same number of incident photons. $d$ is the dilution factor (see Fig. 6), $p_{\odot}$, and $p_{T}$ are the polarization degrees of the circularly polarized photon beam and the target, respectively.

Figure 10 (left) shows the total cross section for $\gamma p \rightarrow$ $\pi^{0} p$ [100] in comparison to some PWA fits. The total cross section is well described by all three models. Also shown are the contributions from anti-parallel $\left(\sigma_{1 / 2}\right)$ and parallel $\left(\sigma_{3 / 2}\right)$ spin settings of photon and proton. It becomes directly obvious by eye, that the resonant contributions to both cross sections are rather different. $\sigma_{3 / 2}$ shows a clear peaking structure not only in the second but also in the 

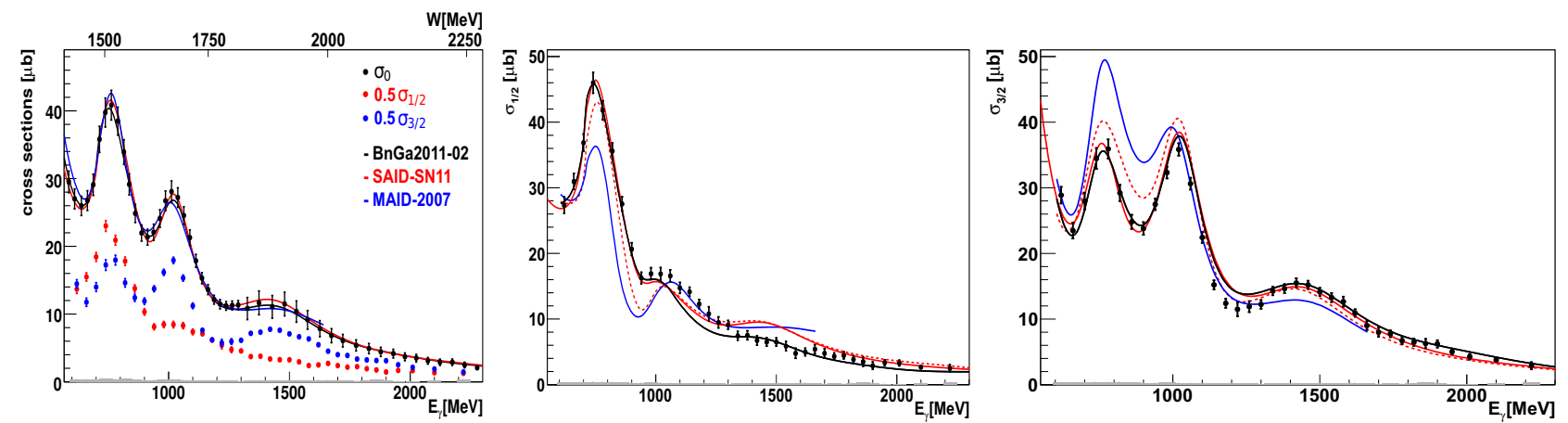

Figure 10. Left: The total cross section $\sigma_{0}$ [71] plotted together with its two helicity components as a function of $E_{\gamma}$. The error bars give the statistical errors, the systematic errors are shown as a dark gray band. $\sigma_{1 / 2}$ (center) and $\sigma_{3 / 2}$ (right) for $\gamma p \rightarrow p \pi^{0}$, PWA predictions: black solid curve: BnGa2011-02, blue solid curve: MAID, red solid curve: CM12, red dashed curve: SN11. For further details see $[100,101]$.

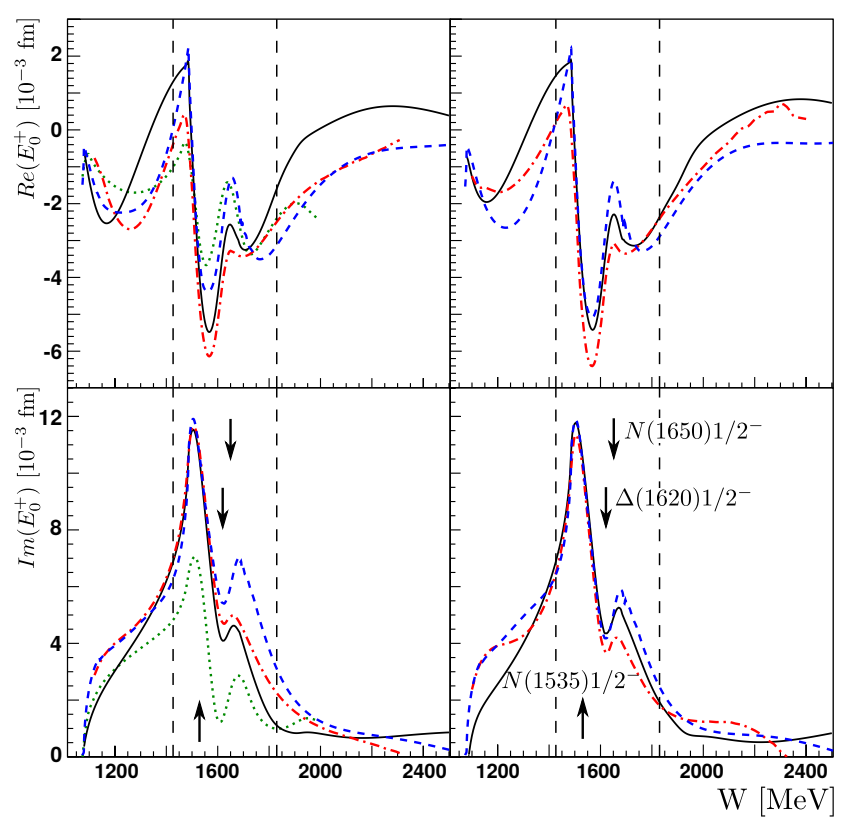

Figure 9. The real and imaginary part of the multipole $E_{0+}$, before the new data were included (left) and after (right), determined by the BnGa PWA (2011-02 [42] resp. 2014-2 [48]: black solid line), the SAID (CM12 [94] resp. new fit [97]: red dashed-dotted line), the JüBo model (2015-B [95] resp. new fit [98]: blue dashed line) and the MAID model (green dotted line) [93]. The dashed lines mark the region covered by the observable $G$. Additionally, the positions of the resonances $N(1535) 1 / 2^{-}$, $N(1650) 1 / 2^{-}$and $\Delta(1620) 1 / 2^{-}$, which contribute to the $E_{0+}$ multipole, are marked with arrows.

third and fourth resonance region, $\sigma_{1 / 2}$ shows only a peak in the second resonance region while only a shoulder in the third and no peaking structure at all is observed in the fourth resonance region. Significant differences are seen when the partial wave analysis predictions are compared to $\sigma_{1 / 2}$ and $\sigma_{3 / 2}$ separately (see Fig. 10, center and right). Even at low energies where one might have expected that everything is well understood based on the existing photoproduction and $\pi N$-elastic scattering data large discrepancies occur. They become even more obvious if the predic-

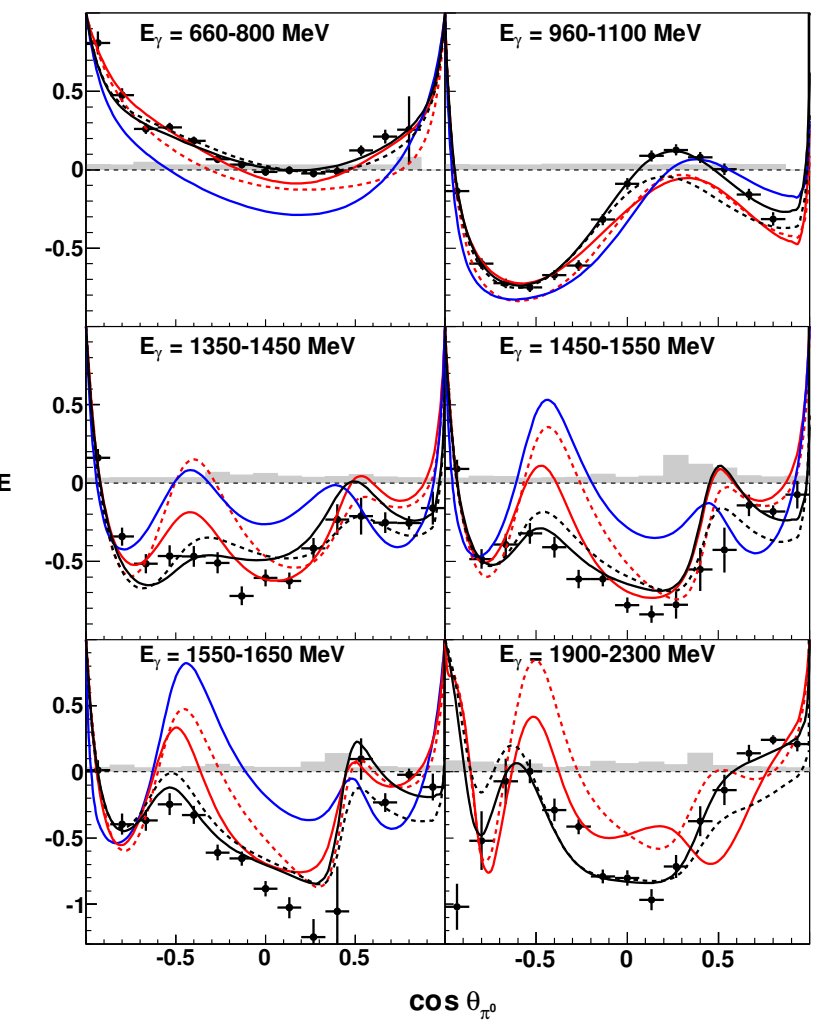

Figure 11. The helicity asymmetry $E$ as a function of $\cos \theta_{\pi}$ for selected $E_{\gamma}$ bins. PWA predictions: black dashed curve: BnGa2011-02, blue solid curve: MAID, red solid curve: CM12, red dashed curve: SN11. Fit to the data points (BnGa2011-02E): black solid curve. Figures taken from [100].

tions are compared to the double-polarization observable $E$ as function of $\cos \left(\theta_{\mathrm{CMS}}^{\pi^{0}}\right)$ (see Fig. 11). None of the partial wave analyses predicted the data precisely. A BnGa refit included these data and gave reasonable consistency between data and fit curve.

The data were included in the multi-channel BnGaPWA and were the basis for a precise determination of the helicity amplitudes of several baryon resonances. Together with our data on $G, T, P$ and $H$, these data led to a determination of the $N(1520) 3 / 2^{-}$helicity amplitudes 
from an energy-independent multipole analysis [102] and to a significantly better determination of the contributing multipole amplitudes (see Fig. 18 in the contribution of project A.2 "Partial Wave Analysis" to these proceedings). Averaged over all multipoles and energies the error bands of the multipoles reduced by a factor 2.25 . This demonstrates the value of the new polarization data taken with the CBELSA/TAPS-experiment at ELSA with polarized photon beams and polarized targets in an impressive way.

\section{$4.4 \gamma p \rightarrow \pi^{0} p$ : The polarization observables $T, P, H$}

The observables $T, P$, and $H$ can be measured simultaneously when data with a transversely polarized target and a linearly polarized photon beam are taken. The measurements [102, 103] were performed using two orthogonal settings of the polarization plane $(\|$ and $\perp)$. The polarization reached its maximum of $\mathrm{p}_{\gamma}=65 \%$ at $850 \mathrm{MeV}$ and dropped down to $40 \%$ at $700 \mathrm{MeV}$. The mean target-proton polarization reached was $\mathrm{p}_{\mathrm{T}} \approx 75 \%$. Data were taken with two opposite settings of the target polarization direction $(\downarrow$ and $\uparrow)$. In that case, the azimuthal distribution of events is given by

$$
\begin{aligned}
\frac{N(\phi)}{N_{0}}=1 & -p_{\gamma} \Sigma_{\text {eff }} \cos (2 \phi)+d p_{T} T \sin (\phi-\alpha) \\
& -d p_{T} p_{\gamma} P \cos (2 \phi) \sin (\phi-\alpha) \\
& +d p_{T} p_{\gamma} H \sin (2 \phi) \cos (\phi-\alpha),
\end{aligned}
$$

where $\alpha$ is the azimuthal angle between the target polarization vector and the photon polarization plane. $T, P$, and $H$ are determined, for each $\left(E_{\gamma}, \cos \theta_{\pi^{0}}\right)$ bin, by fits to the measured azimuthal distribution of events. $T$ is determined from a fit to the azimuthal yield asymmetry:

$$
\Delta N(\phi)_{\mathrm{T}}=\frac{1}{d \cdot \mathrm{p}_{\mathrm{T}}} \cdot \frac{N_{\uparrow}-N_{\downarrow}}{N_{\uparrow}+N_{\downarrow}}=T \cdot \sin (\beta-\phi)
$$

A typical example for such a fit is shown in Fig. 13, left panel. $P$ and $H$ are extracted from data where not only the target polarization is changed but also the photon polarization plane from $\|$ to $\perp$, using the equation:

$$
\begin{gathered}
\Delta N(\phi)_{\mathrm{BT}}=\frac{1}{d \cdot \mathrm{p}_{\gamma} \mathrm{p}_{\mathrm{T}}} \cdot \frac{\left(N_{\perp \uparrow}-N_{\perp \downarrow}\right)-\left(N_{\| \uparrow}-N_{\| \downarrow}\right)}{\left(N_{\perp \uparrow}+N_{\perp \downarrow}\right)+\left(N_{\| \uparrow}+N_{\| \downarrow}\right)} \\
=P \sin (\beta-\phi) \cos (2(\alpha-\phi))-H \cos (\beta-\phi) \sin (2(\alpha-\phi))
\end{gathered}
$$
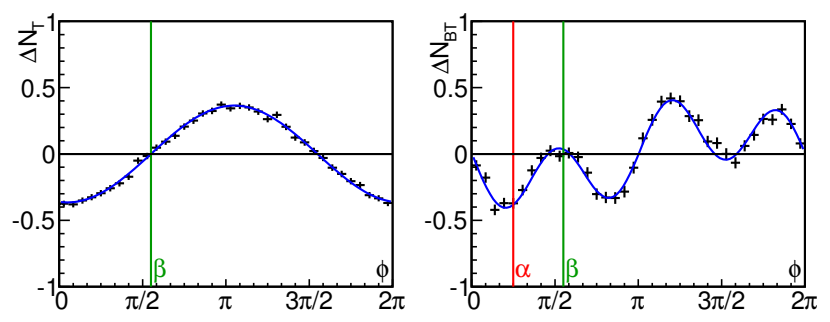

Figure 13. Event yield asymmetry as a function of $\phi$, left: $\Delta N(\phi)_{\mathrm{T}}$, right: $\Delta N(\phi)_{\mathrm{BT}}$, fitted by the functions given in eqs. (6) and (7), respectively. Shown is the energy bin $W=1.524-$ $1.542 \mathrm{GeV}$ as example [103].
The observables $P$ and $H$ are determined by a fit to the

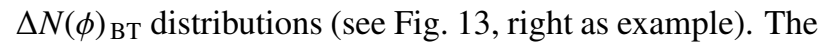
polarization observables $T, P$, and $H$ are shown in Fig. refpic:tph for selected bins. The lower energy data (up to $\mathrm{W}=$ $1620 \mathrm{MeV}$ ) were used as basis for an energy-independent trucated partial wave analysis in sliced energy bins (see the discussion in project A.2 "Partial Wave Analysis" and in [102]). In addition to the data on $T, P$, and $H$, differential cross section data (e.g. [71]), data on $\Sigma$ [90, 91, 96], $G$ [86] and on $E$ [100] were included for this reaction, making in total seven observables available for the PWA. It should be mentioned that this was the first time that the available data allowed for an energy-independent reconstruction of multipoles in the energy range covering the second resonance region of $N(1520) 3 / 2^{-}$and $N(1535) 1 / 2^{-}$. Based on this analysis, the helicity amplitudes of $N(1520) 3 / 2^{-}$were deduced with minimal model assumptions [102]. The result is inconsistent at the level of more than $2 \sigma$ with older (model-dependent) determinations and supports those of the BnGa PWA.

\section{$4.5 \gamma p \rightarrow p \pi^{0}:$ An expansion into partial waves}

It is desirable to have simple methods that help to infer some of the physics contained in newly measured polarization data, without having to do a full energy-dependent partial wave analysis (see project A.2 "Partial Wave Analysis"). The ansatz described here is capable of giving a good estimate of the maximal angular momentum content $\mathrm{L}_{\max }$ needed to describe a given data set in any kind of partial wave analysis. More details on the method, as well as its application to a wide array of polarization data, will be published in a forthcoming paper [105].

The model-independent decomposition of the full $T$ matrix of pseudoscalar meson photoproduction into, for instance, four CGLN [107] spin-amplitudes $F_{i}$ and the expansion of these amplitudes into electric $\left(E_{\ell \pm}\right)$ and magnetic $\left(M_{\ell_{ \pm}}\right)$multipoles is well-known. In single-meson photoproduction, 16 polarization observables (including the unpolarized cross section) are measurable (see Table 3 ). In case the multipole expansion is combined with a consistent definition of the 16 polarization observables in terms of CGLN-amplitudes [108], one arrives at a polynomial parametrization for the angular distributions of the profile functions. Here for example, the parametrization is given for the profile function $\check{T}$ of the target asymmetry $T$

$$
\begin{aligned}
\check{T}(W, \theta) & =T \cdot\left(\frac{d \sigma}{d \Omega}\right)_{0} \\
& =\rho \sum_{n=1}^{2 \mathrm{~L}_{\max }}\left(a_{\mathrm{L}_{\max }}\right)_{n}^{\check{T}}(W) P_{n}^{1}(\cos \theta) .
\end{aligned}
$$

The phase-space factor $\rho:=q / k$ was defined, with $k$ and $q$ being the moduli of the CMS-three-momenta of the initial photon and final state meson. Finally, $P_{n}^{1}$ are the associated Legendre polynomials [109], $P_{n}^{m}$ with $m=1$. It has to be noted, that the upper index $m$ depends on the parametrized observable. For the profile functions $\breve{E}$ and $\breve{G}$, for instance, 


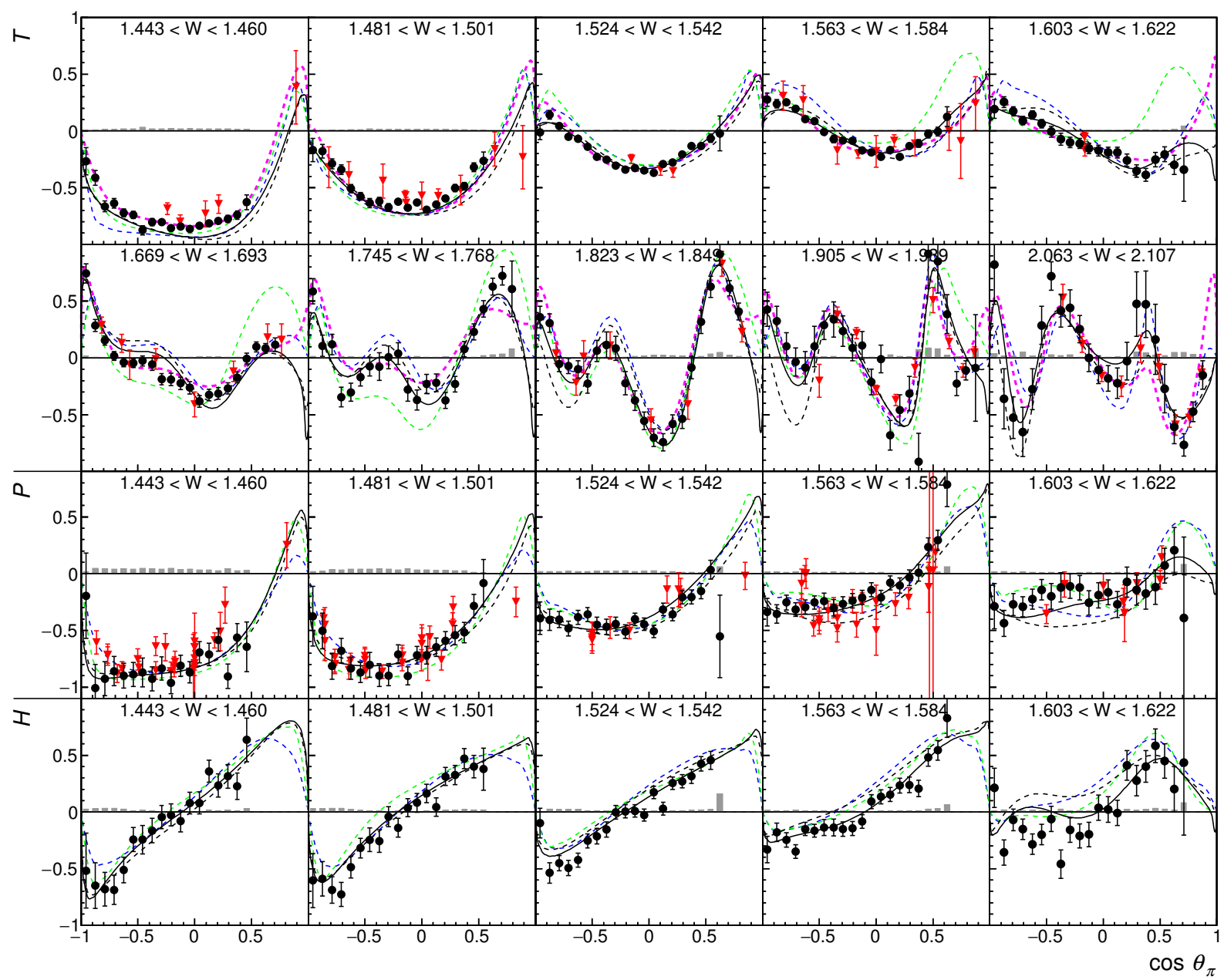

Figure 12. Few selected bins for the polarization observables $T, P$, and $H$ for the reaction $\gamma p \rightarrow p \pi^{0}$ as function of the $\gamma p$ invariant mass $W$ (in $\mathrm{GeV}$ ) and of the scattering angle $\cos \theta$. The systematic error is shown as dark gray band. An additional systematic error on the photon energy which reaches from $\sigma_{E_{\gamma}}^{\text {sys }}=6.5 \mathrm{MeV}$ at the lowest to $2.3 \mathrm{MeV}$ at the highest energy bin plotted is not shown. The low energy data were presented in $[102,103]$. Earlier data (red triangles) are from [104]. The solid line represents our best fit (BnGa2014). The data are compared to predictions (dashed curves) from BnGa2011-02 (black), MAID (light green), and SAID CM12 (blue).

the precise form of the parametrization similar to (8) reads

$$
\begin{aligned}
& \check{E}(W, \theta)=\rho \sum_{n=0}^{2 \mathrm{~L}_{\max }}\left(a_{\mathrm{L}_{\max }}\right)_{n}^{\check{E}}(W) P_{n}^{0}(\cos \theta), \\
& \check{G}(W, \theta)=\rho \sum_{n=2}^{2 \mathrm{~L}_{\max }}\left(a_{\mathrm{L}_{\max }}\right)_{n}^{\check{G}}(W) P_{n}^{2}(\cos \theta) .
\end{aligned}
$$

The Legendre coefficients $a_{\mathrm{L}_{\max }}$ are themselves bilinear Hermitian forms in the multipoles. In case of $\check{T}$, one can express the Legendre coefficients as

$$
\left(a_{\mathrm{L}_{\max }}\right)_{n}^{\check{T}}(W)=\left\langle\mathcal{M}_{\mathrm{L}_{\max }}(W)\left|\mathcal{C}_{n}^{\check{T}}\right| \mathcal{M}_{\mathrm{L}_{\max }}(W)\right\rangle,
$$

defined by certain (calculable) matrices $C_{n}^{\check{T}}$ of dimension $4 \mathrm{~L}_{\max } \times 4 \mathrm{~L}_{\max }$ and the multipoles $\mathcal{M}_{\mathrm{L}_{\max }}$ [105]. The multipoles are here written as the $4 \mathrm{~L}_{\max }$-dimensional complex vector

$$
\begin{aligned}
\left|\mathcal{M}_{\mathrm{L}_{\max }}\right\rangle= & {\left[E_{0+}, E_{1+}, M_{1+}, M_{1-}, E_{2+}, E_{2-}, M_{2+}, M_{2-},\right.} \\
& \left.\ldots, E_{\mathrm{L}_{\max }}, E_{\mathrm{L}_{\max }-}, M_{\mathrm{L}_{\max }}, M_{\mathrm{L}_{\max }}\right]^{T}
\end{aligned}
$$

which is defined for any $\mathrm{L}_{\max } \geq 1$. The maximal partial wave content needed to describe an observable can now be deduced. One usually starts at $\mathrm{L}_{\max }=1$, performs a simple $\chi^{2}$-test for a fit to the data with a parametrization such as (8). The angular momentum $\mathrm{L}_{\max }$ is increased in case the test is not passed, i.e. until $\chi^{2} /$ ndf $\simeq 1$ is fulfilled. This procedure is continued up to the lowest order $\mathrm{L}_{\max }$ where the $\chi^{2}$-test suggests a good fit. Then, this method directly returns an estimate for the maximal angular momentum content needed. Interesting phenomena can be seen drawing the $\chi^{2} / \mathrm{n}_{\mathrm{df}}$ of each fitted order $\mathrm{L}_{\max } \geq 1$ against the center-of-mass energy $W$. Such plots are shown in Figs. 14,15 , and 16 for $G$ [86, 87], $E$ [101], and $T$ [102, 103]. For specific truncation orders, bumps can be observed in the $\chi^{2}$-plots within certain energy regions. These bumps can be cautiously attributed to well-known resonances (see Fig. 1). For more details, see reference [105].

The evaluation of the matrices defining bilinears such as (11), which are calculable, opens the door to the comparison of the fitted Legendre coefficients to energy dependent partial wave analysis models. In this case here, the Bonn-Gatchina solution BnGa 2014-02 was chosen 

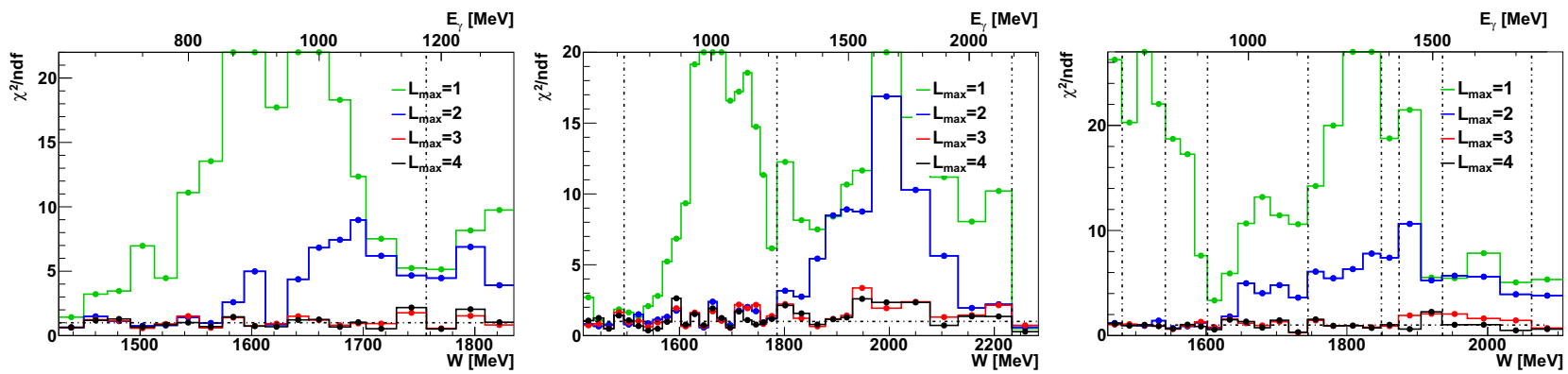

Figure 14. The $\chi^{2} / n_{\mathrm{df}}$ values of fits with associated Legendre polynomials according to eq. (8) to the recent new double polarization observable $\breve{G}[86,87]$ (left) $\check{E}[100,101]$ (center), and $\check{T}[102,103]$ (right), Only statistical errors were used in the fits. The partial wave expansion was truncated at $\mathrm{L}_{\max }=1 \ldots 4$. The $\chi^{2} / n_{\mathrm{df}}$ values are plotted for fits with different $\mathrm{L}_{\max }$ as a function of the center of mass energy W. Results are preliminary and will be published in [105].
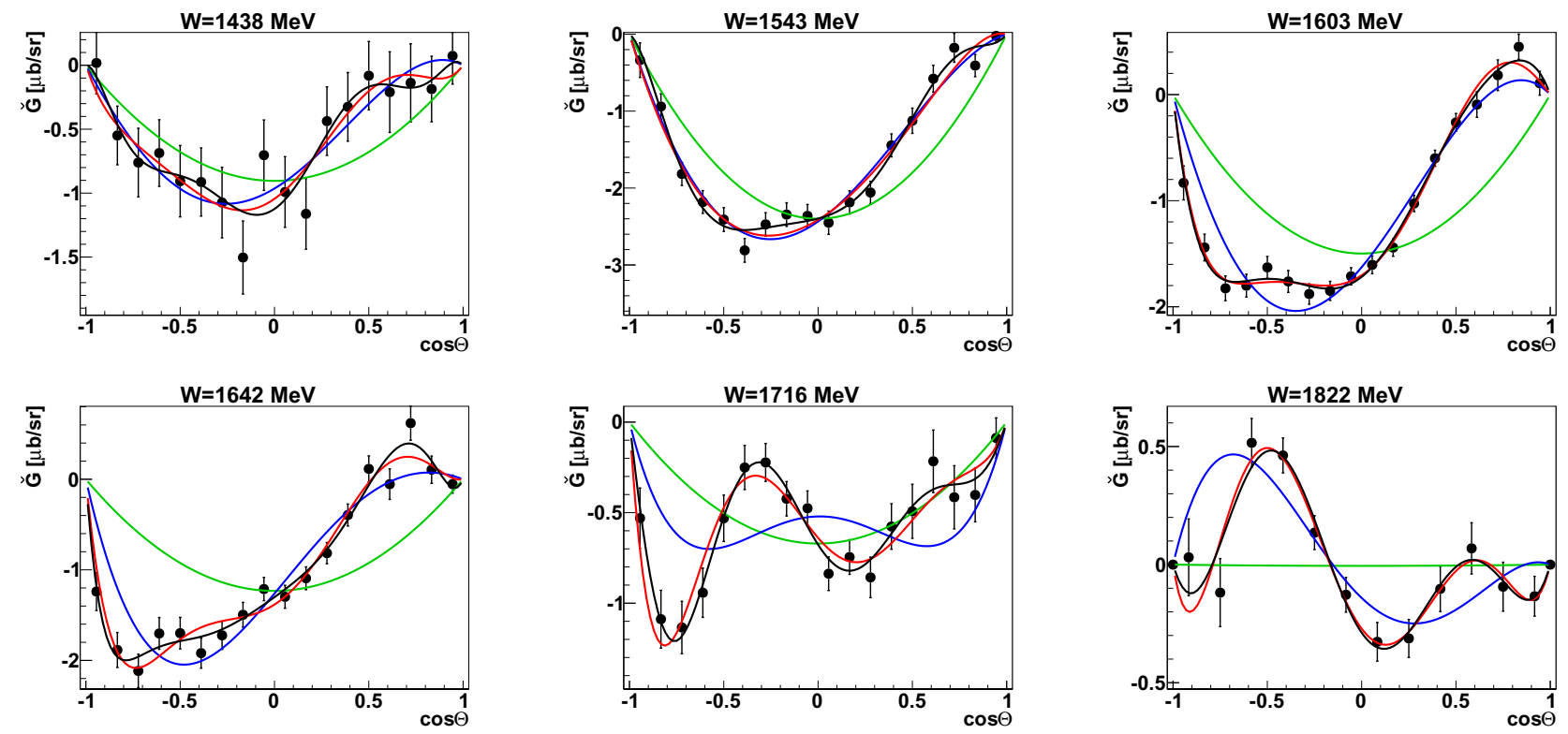

Figure 15. 6 out of 19 selected angular distributions of $\breve{G}$ (black points) with the different $\mathrm{L}_{\max }$ fits (solid lines) in the range from $W=1438 \mathrm{MeV}$ to $1822 \mathrm{MeV}$. Colors are the same as in Fig. 14.
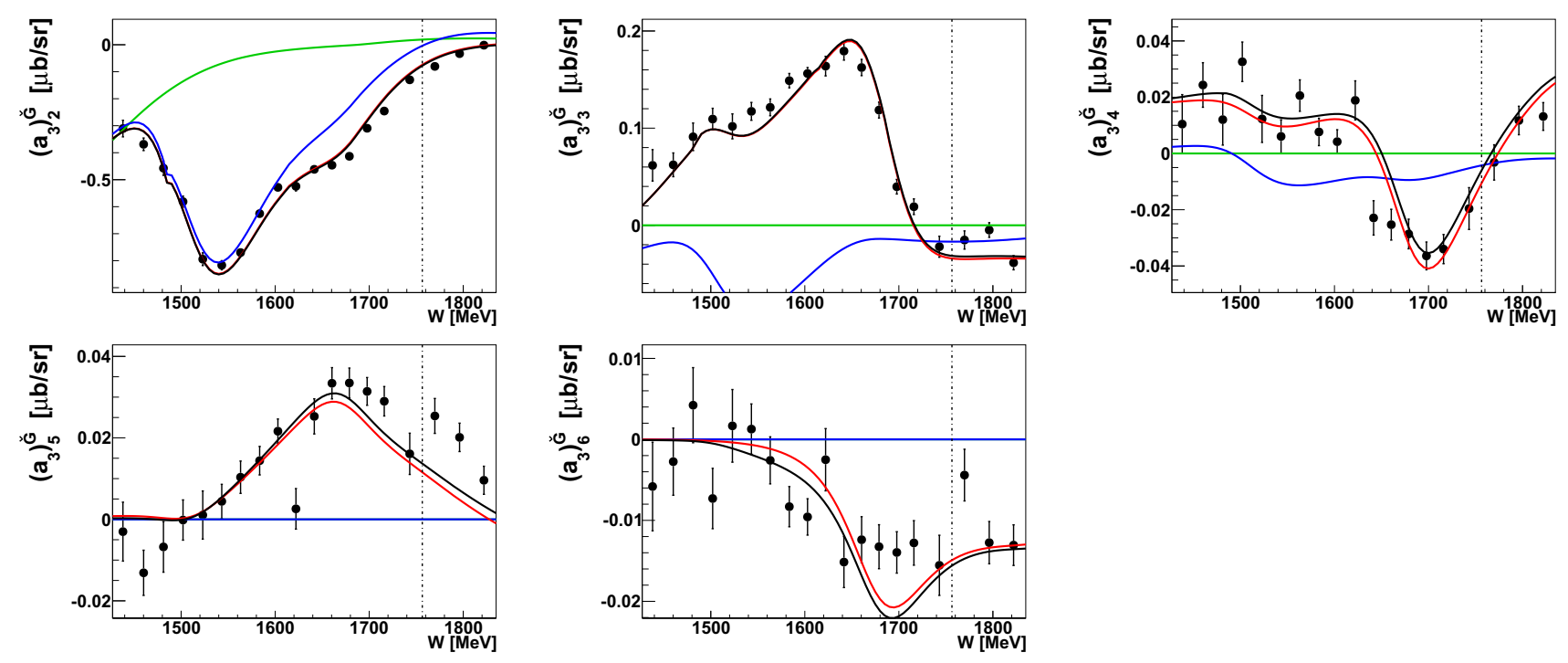

Figure 16. Comparison of the fit coefficients for $\mathrm{L}_{\max }=3$ (black points), $\left(a_{3}\right)_{2 \ldots 6}^{\check{G}}$ (see eq. 10), with the BnGa2014-02 solution truncated at different $\mathrm{L}_{\max }$ (solid lines). Colors are the same as in Fig. 14 . 
(see project A.2). Then, the $a_{\mathrm{L}_{\max }}$ evaluated from modelmultipoles up to a certain order can be plotted in comparison to the fitted values. This has been done for the $G$ observable in Figure 16. Then, the Legendre coefficients can help us to learn about the influence of the contributing multipoles, at least in the context of the employed model.

The $\chi^{2}$-plot in Fig. 14 suggests a good description using $D$-waves for energies below $W=1600 \mathrm{MeV}$ for $T$, $E$, and $G$. For higher energies, all the $\chi^{2}$-plots consistently enforce at least $F$-waves, since $\chi^{2} / \mathrm{n}_{\mathrm{df}}$ becomes larger than 1 for the $\mathrm{L}_{\max }=2$-fit. This behavior is well-reflected in the coefficient plot for $\left(a_{3}\right)_{2}^{\breve{G}}$. However, the $F$-waves from Bonn-Gatchina are needed to reproduce $\left(a_{3}\right)_{3}^{\breve{G}}$ for all energies and therefore in particular also in the lower energy region. This is an example of an interference phenomenon. There can be interferences between dominant lower and even small higher partial waves, taking place in the lower Legendre coefficients, to which the simple method described here is insensitive.

An important lesson learned from model comparisons such as these is the fact that the data needs to be precise enough to be able to detect also the interferences of large resonant multipoles with small background partial waves. Quite generally, Legendre coefficients are well suited for model comparisons, since here effects can become apparent which are practically invisible if one compares just the angular distributions of the data to a model. Apart from the above-mentioned model-comparison, the investigation of Legendre coefficients is also useful for the comparison of different data sets. In particular, the size and influence of different systematic effects in the data can become more visible. As an outlook, in the case the data shall be processed further in a truncated partial wave analysis (TPWA), one can get a first good idea about the cutoff $\mathrm{L}_{\max }$ from in this kind of analysis.

\section{6 $\gamma p \rightarrow \eta p$ : The polarization observables $E, G, T$, $\boldsymbol{P}, \boldsymbol{H}$}

New single and double polarization observables were also determined for $\gamma p \rightarrow p \eta$ [110]. The observables $E$ [111], $G$ [112], $T, P$, and $H$ [113] have been measured with the CBELSA/TAPS experiment. Data on $T$ and $F$ [114] were obtained in Mainz, and data on $E$ have recently been published by CLAS [115]. The latter data are consistent with our CBELSA/TAPS-data but are not yet included in the BnGa-PWA.

A few bins of the newly measured double polarization observables are shown in Figs. 17-19 for $E, T, P$, $H$, and $G$, together with the predictions from different PWAs. None of the predictions comes even close to the data, even though they do describe all the so far existing $\eta$-photoproduction data $(\mathrm{d} \sigma / \mathrm{d} \Omega, \Sigma)$ reasonably well. This clearly shows that the amplitudes for $\eta$-photoproduction were even worse constrained by existing data than for $\pi^{0}$ photoproduction. Of course this was to be expected since $\pi N$-elastic scattering provides already significant information on the $\pi N$ coupling of resonances, while data of comparable accuracy is missing for the $\eta N$-final state. Data on $\pi N \rightarrow \eta N$ are scarce.

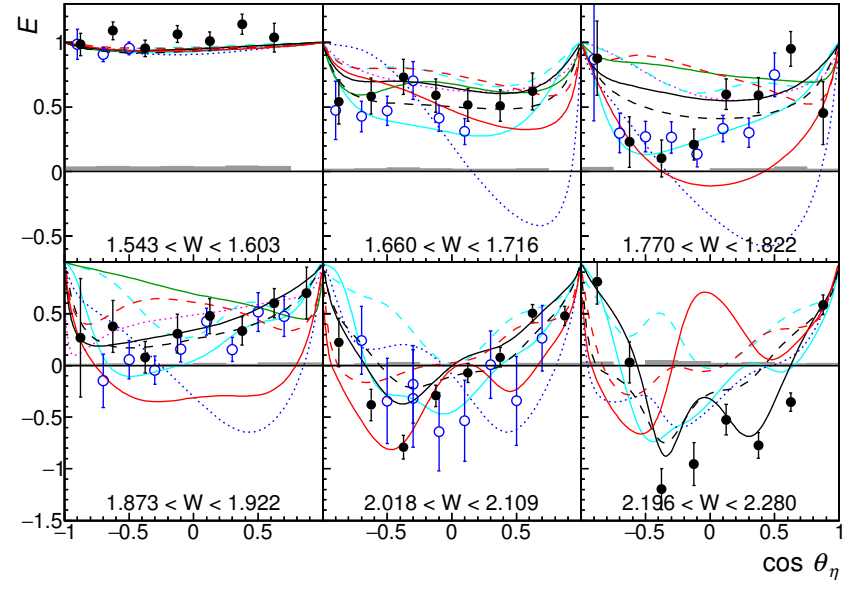

Figure 17. CBELSA/TAPS data on the polarization observable $E$ (only selected bins shown) for $\gamma p \rightarrow p \eta$ (black) plotted together with the respective data from CLAS [115] (blue, open circles) and the predictions from MAID (green), SAID (blue, dotted), JüBo2015 (2015-3) (cyan, dashed (solid)), and BnGa201101(02) (red, solid (dashed)). New BnGa-Fits are shown in black: with (solid) and without (dashed) a new 5/2--state at $2200 \mathrm{MeV}$ included.

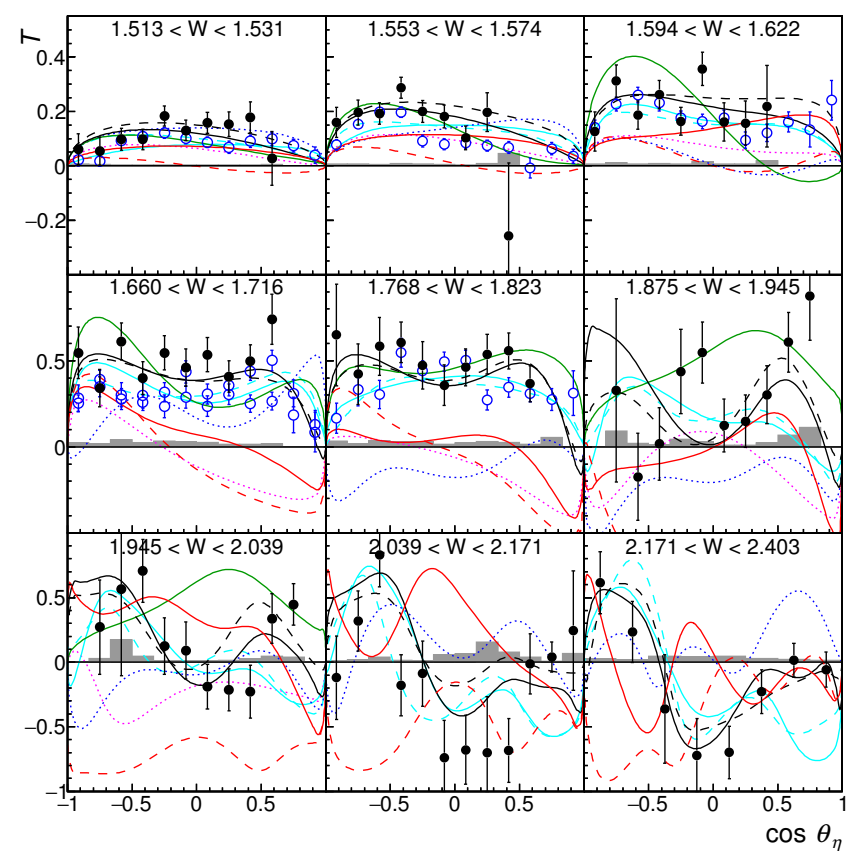

Figure 18. CBELSA/TAPS data on the polarization observable $T$ (only selected bins shown) for $\gamma p \rightarrow p \eta$ (black) plotted together with the respective data from MAMI [114] (blue, open circles). For an explanation of the curves see Fig.17.

The new polarization data on $E, T, P, H, G, F$ (except those from [115]) were included into the BnGa-PWA. Some of the results are presented in Table 4. Further results on other resonances are given in [110]. The $N \eta$ branching ratio found in this new PWA reduces the large and controversially discussed difference in $N \eta$-branching ratios of the $N(1535) 1 / 2^{-}$and the $N(1650) 1 / 2^{-}$significantly. The unexpectedly large $N \eta$-branching ratio difference observed before had led to a large number of very different explanations. Isgur and Karl [12] explained the observed difference in the $p \eta$-coupling by mixing of 

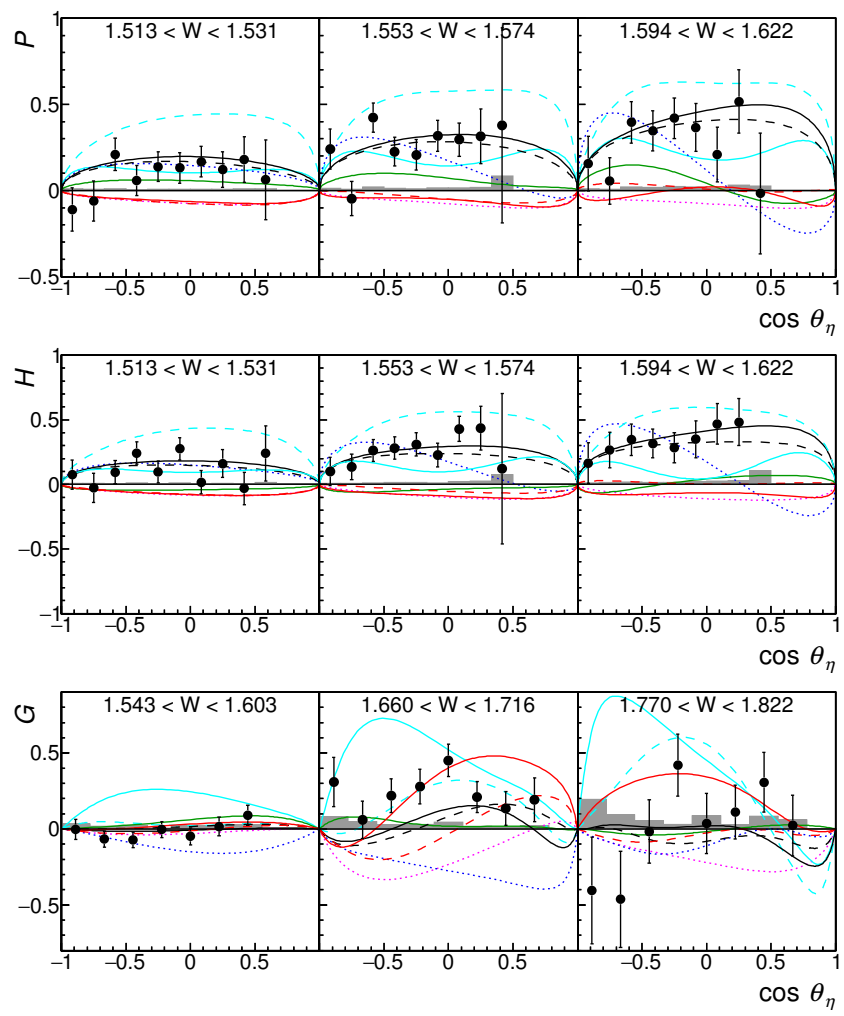

Figure 19. CBELSA/TAPS data on the polarization observable $P$ (top), $H$ (middle), and $G$ (bottom) for $\gamma p \rightarrow p \eta$ (only selected bins shown). For an explanation of the curves see Fig.17.

the two quark-model states with defined total quark spin $S=1 / 2$ and $S=3 / 2$; Kaiser, Siegel and Weise [116] and Mai, Bruns, and Meißner [117] generated N(1535)1/2dynamically (see also project B.3 "Chiral dynamics with (non)strange quarks"). In [116] it is interpreted it as quasi-bound $K \Sigma-K \Lambda$-state decaying strongly into $N \eta$ due to coupled channel effects; Zou [118] assigned a large pentaquark component to the $N(1535) 1 / 2^{-}$wave function.

The new result seems to be at variance with these explanations. The branching ratios given in Table 4 are derived based on the pole position properties of the resonances (for more details see contribution of project A. 2 to these proceedings and [110]). One interesting observation to note, is that the $K$-matrix-coupling to $p \eta$ at the lower

Table 4. Branching ratios BR for $N^{*} \rightarrow N \eta$ decays and the photon helicity amplitudes $A_{1 / 2}, A_{3 / 2}$ for 4 of the contributing nucleon resonances, derived from their properties at the pole position. The helicity amplitudes are given in units of $\mathrm{GeV}^{-1 / 2}$ (taken from [110]). Small numbers below the BRs or below the helicity amplitudes give either the RPP [58] estimate or the entries reported in [42].

\begin{tabular}{|cc|cc|}
\hline \hline Res. & $\mathrm{BR}\left(N^{*} \rightarrow N \eta\right)$ & Res. & $\mathrm{BR}\left(N^{*} \rightarrow N \eta\right)$ \\
$A_{1 / 2}$ & $A_{3 / 2}$ & $A_{1 / 2}$ & $A_{3 / 2}$ \\
\hline$N(1535)$ & $0.42 \pm 0.04$ & $N(1710)$ & $0.25 \pm 0.09$ \\
$1 / 2^{-}$ & $0.42 \pm 0.10$ & $1 / 2^{+}$ & $0.10-0.30$ \\
$0.093 \pm 0.009$ & - & $0.040 \pm 0.020$ & - \\
$0.115 \pm 0.015$ & - & $0.035 \pm 0.012$ & - \\
\hline$N(1650)$ & $0.32 \pm 0.04$ & $N(1720)$ & $0.03 \pm 0.02$ \\
$1 / 2^{-}$ & $0.05-0.15$ & $3 / 2^{+}$ & $0.021 \pm 0.014$ \\
$0.032 \pm 0.006$ & - & $0.115 \pm 0.045$ & $0.135 \pm 0.040$ \\
$0.045 \pm 0.010$ & - & $0.100 \pm 0.020$ & $0.150 \pm 0.030$ \\
\hline \hline
\end{tabular}

mass $K$-matrix pole is large while the one at the higher mass (close to $1650 \mathrm{MeV}$ ) is almost negligible.

An improved description of the new data on $T$ and $E$ is reached if a further high mass $N(2200) 5 / 2^{-}$is included in the fits (see Figs. 17 and 18). Since no convincing evidence for the state was observed in the other reactions one must consider this new resonance with some caution. Of course one would like to see the results of the $\mathrm{BnGa}$ PWA confirmed by other analyses including the new data. Such an analysis is for instance planned within the JüBo approach.

\section{Photoproduction of meson pairs}

\subsection{Differential cross section and measurements with linearly polarized beam}

Data on the reactions $\gamma p \rightarrow p \pi^{0} \pi^{0}$ and $\gamma p \rightarrow p \pi^{0} \eta$ were acquired in different run periods with unpolarized (985,000 $p \pi^{0} \pi^{0}$ and $145,000 p \pi^{0} \eta$ events) and with linearly polarized photons $(620,000 / 105,500$ events). Figure 20 shows two Dalitz plots for two-pion production, for $1500<E_{\gamma}<1700 \mathrm{MeV}$ and for $1800<E_{\gamma}<2200 \mathrm{MeV}$. Prominent structures in the distributions can be seen: at the lower photon energy, $\Delta(1232)$ and $N(1520) 3 / 2^{-}$are produced as intermediate isobar in a sequential decay chain. At the higher photon energy, $N(1680) 5 / 2^{+}$shows up in addition. In the reaction $\gamma p \rightarrow p \pi^{0} \eta, N(1535) 1 / 2^{-}$is seen above the $p \eta$ threshold (see Fig. 21). With increasing photon energy, the $\Delta(1232) \eta$ isobar can be observed as well as contributions from $p a_{0}(980)$.

\section{$5.2 \vec{\gamma} p \rightarrow p \pi^{0} \eta$ : First measurement of the polariza- tion observables $I^{s}$ and $I^{c}$ in photoproduction of two mesons}

Using linear photon polarization, new observables show up, called $I^{s}$ and $I^{c}$, which have been determined for the first time in [47]. Before [47] two-meson photoproduction using linearly polarized photons has been treated in a quasi two-body approach only resulting in the extraction of the beam asymmetry $\Sigma[45,51,119]$ known from singlemeson photoproduction. In fact, however, three-body final states like $p \pi^{0} \eta$ or $p \pi^{0} \pi^{0}$ yield additional degrees of freedom, reflected in a different set of polarization observables [63], which can be accessed in a full three-body approach. The cross-section for the photoproduction of pairs of pseudoscalar mesons, disregarding target- and recoil polarization, is given by:

$$
\frac{\mathrm{d} \sigma}{\mathrm{d} \Omega}=\frac{\mathrm{d} \sigma_{0}}{\mathrm{~d} \Omega}\left[1+\delta_{\odot} I^{\odot}\left(\phi^{*}\right)+\delta_{l}\left(I^{c}\left(\phi^{*}\right) \cos 2 \phi+I^{s}\left(\phi^{*}\right) \sin 2 \phi\right)\right] .
$$

Here $\frac{\mathrm{d} \sigma_{0}}{\mathrm{~d} \Omega}$ denotes the unpolarized cross-section and $\delta_{\odot}\left(\delta_{l}\right)$ the degree of circular (linear) polarization. The polarization observables $I^{s}, I^{c}$, and $I^{\odot}$ emerge as the amplitudes of the respective modulations of the azimuthal distributions of the final state particles (Fig. 22, right). These occur if the non-coplanar kinematics of the reaction is considered by taking into account the angle $\phi^{*}$ between the reaction plane (spanned by the incoming photon and one recoiling particle) and the decay plane (comprising all three final 

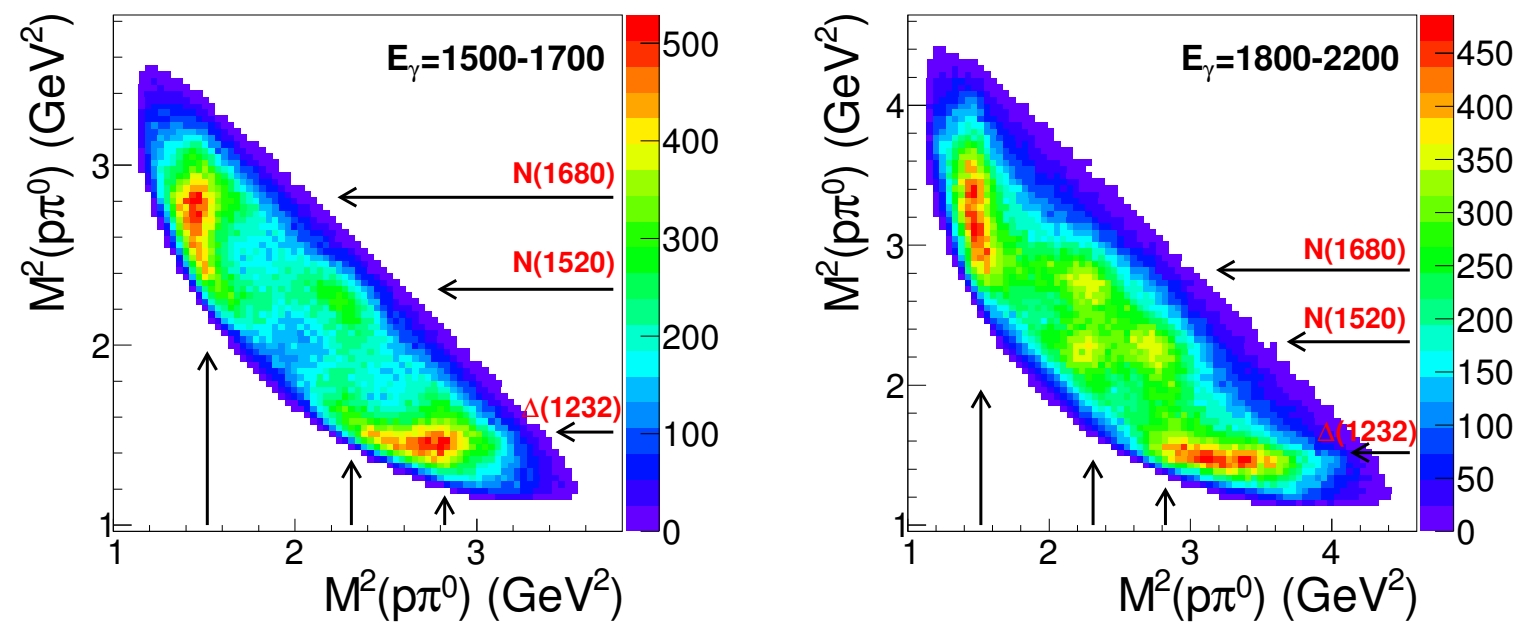

Figure 20. Dalitz plots for $1500 \mathrm{MeV}<\mathrm{E}_{\gamma}<1700 \mathrm{MeV}$ (left) and $1800 \mathrm{MeV}<\mathrm{E}_{\gamma}<2200 \mathrm{MeV}$. The arrows indicate the positions of $\Delta(1232), N(1520) 3 / 2^{-}$, and $N(1680) 5 / 2^{+}$.
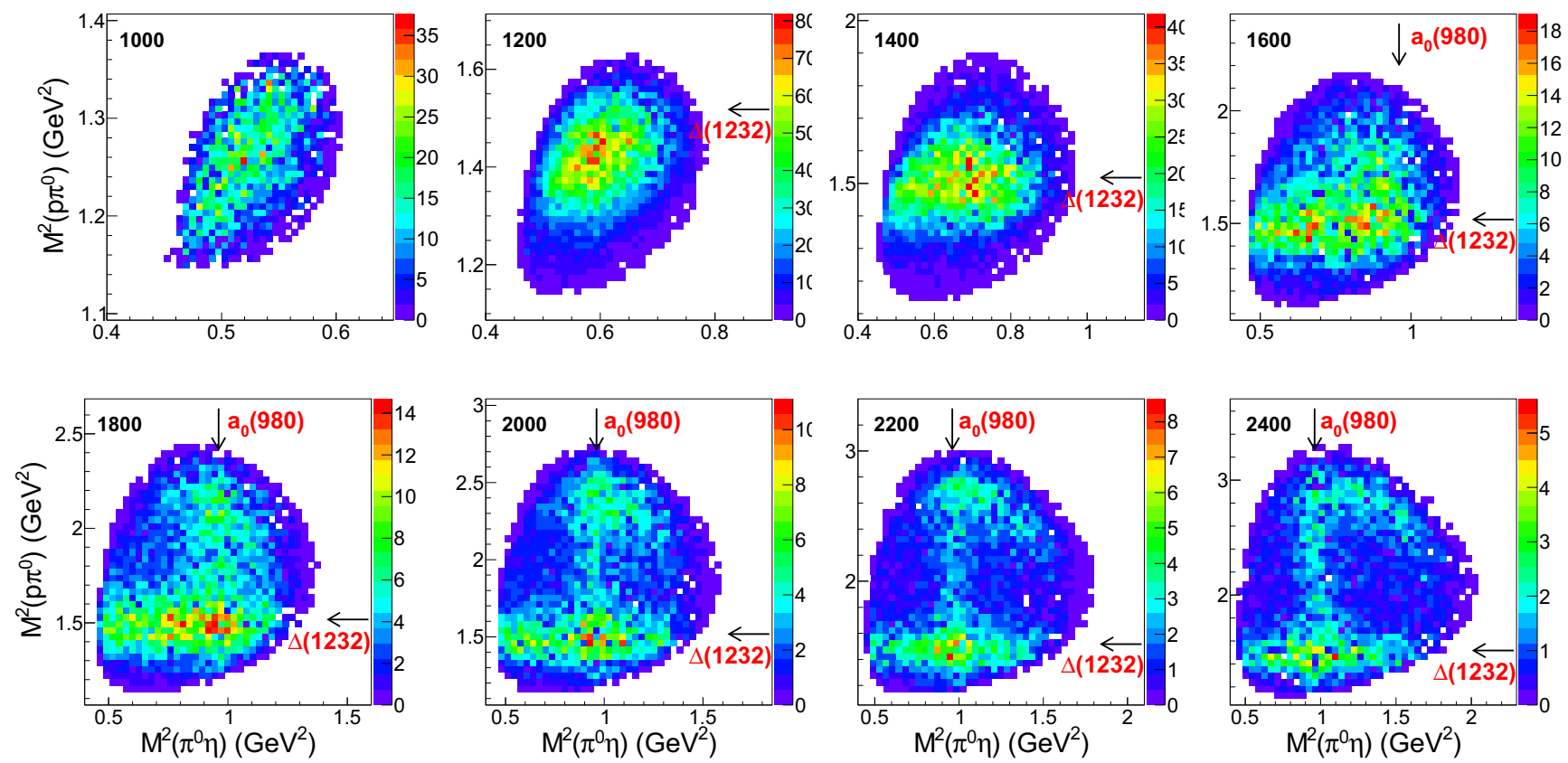

Figure 21. Dalitz plots $M^{2}(p \eta)$ versus $M^{2}\left(\pi^{0} \eta\right)$ for the incoming photon energy ranges $1000 \pm 100 \mathrm{MeV}$ to $2400 \pm 100 \mathrm{MeV}$.

state particles) (Fig. 22, left). If intergrated over $\phi^{*}, I^{s}$ and $I^{\odot}$ vanish, while $I^{c}$ transfers into the beam asymmetry $\Sigma$, well known for two-particle final states.

The full three-body approach with linearly polarized photons was applied for the first time for the reaction $\gamma p \rightarrow p \pi^{0} \eta[47,48]$. This reaction allows us to study highlying $\Delta$-resonances via their isospin-selective $\Delta(1232) \eta$ decay. $\Delta(1700) 3 / 2^{-}$and $\Delta(1940) 3 / 2^{-}$were thus identified. The latter observation led to an upgrade of the $\Delta(1940) 3 / 2^{-}$resonance from a one-star to a two-star resonance in the RPP [14]. In addition, the couplings of resonances to $p a_{0}(980)$ were investigated. Evidence for the corresponding intermediate states is seen in Fig. 23, top. Also the $N(1535) 1 / 2^{-} \pi$ intermediate state was observed.
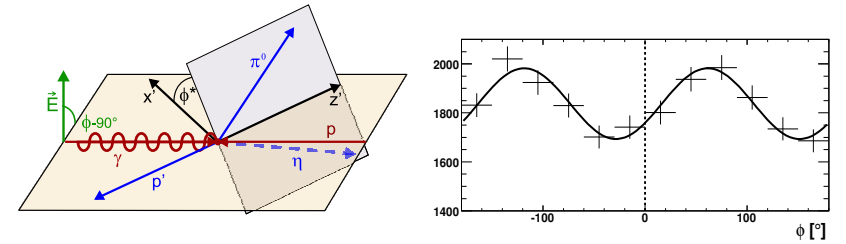

Figure 22. Left: Definition of the relevant angles in a three-body final state. Right: Example for a measured $\phi$-distribution for a limited $\phi^{*}$ range $\left(\gamma p \rightarrow p \pi^{0} \pi^{0}, 970 \mathrm{MeV}<E_{\gamma}<1200 \mathrm{MeV}, \phi^{*}: 18-36^{\circ}\right)$. Here, both the $\cos 2 \phi$ - as well as the $\sin 2 \phi$-modulation (as a phase-shift) of the cross-section are visible.

Examples for the extracted asymmetries $I^{S}$ and $I^{c}$ are shown in Fig. 23 (bottom). The data on $I^{s}$ and $I^{c}$ 
are compared to three theoretical models, to an early BnGa-PWA [43, 44], to the analysis by Fix et al. [120, 121], neglecting $p a_{0}$-couplings and assuming $\Delta(1232) \eta$ dominance in most resonance decays, and Döring et al. [122], describing the reaction by the excitation of the $\Delta(1700) 3 / 2^{-}$resonance, which in this model is dynamically generated. Close to threshold all models are able to reproduce the observables reasonably well. In the higherenergy region, shown in Fig. 23 (bottom), only the BnGaPWA, including $\Delta(1232) \eta, p a_{0}(980)$ and $N(1535) 1 / 2^{-} \pi$ decays (as well as excited isospin $I=1 / 2$ states) gives a reasonable description of the data. This demonstrates the high sensitivity of the observables $I^{s}$ and $I^{c}$ to the contributing resonances and their decays.

\section{$5.3 \vec{\gamma} p \rightarrow p \pi^{0} \pi^{0}: I^{S}$ and $I^{c}$ in $2 \pi^{0}$-photoproduction}

Also in the photoproduction of two neutral pions, the observables $I^{s}$ and $I^{c}$ exhibit a high sensitivity to the reaction dynamics. Fig. 24 (top) shows the Dalitz plot for the reaction exhibiting various intermediate resonant structures.

In the analysis of our data on $\gamma p \rightarrow p \pi^{0} \pi^{0}[53,54]$ with no photon polarization two equally good descriptions of the data were obtained within the BnGa-PWA. One solution yielded $S$-wave dominance in the decay
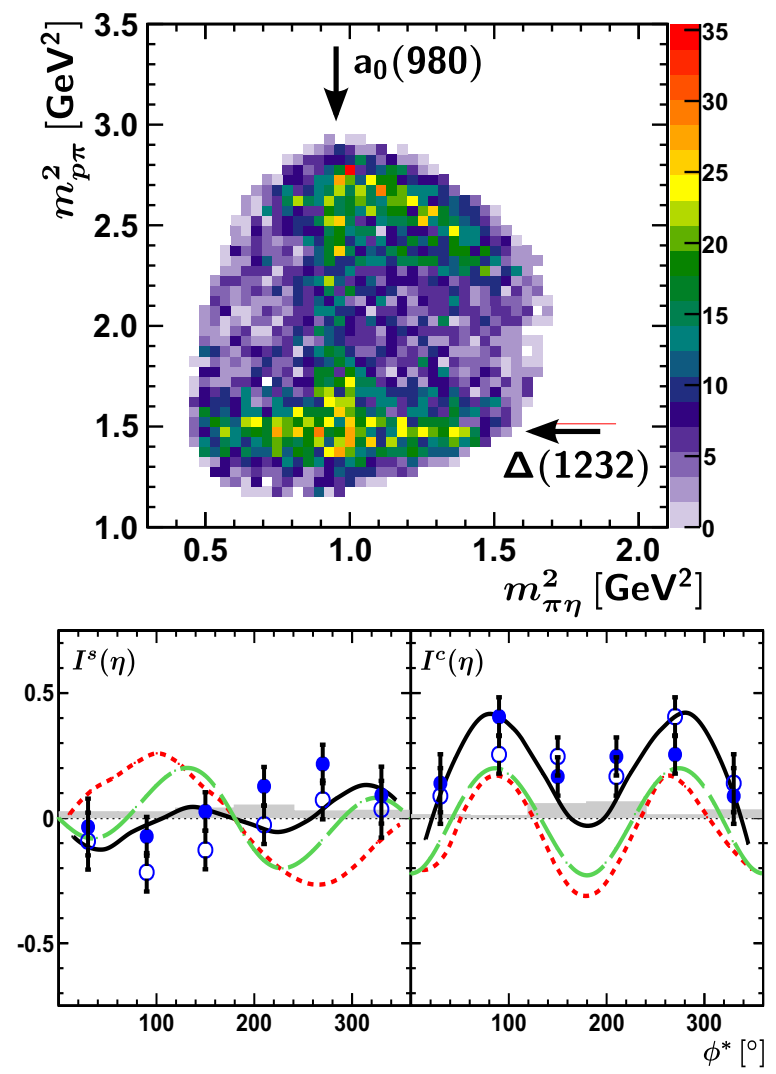

Figure 23. Top: $m^{2}\left(p \pi^{0}\right)-m^{2}\left(\pi^{0} \eta\right)$ Dalitz plot for $E_{\gamma}=2150 \pm 50 \mathrm{MeV}$. Marked are the signatures of the $\Delta(1232) 3 / 2^{+}$and the scalar meson $a_{0}(980)$, additionally, the signal for the $N(1535) 1 / 2^{-}$is visible in the diagonal. Bottom: Exemplary distributions for the observables $I^{s}$ and $I^{c}$ ( $\mathrm{W}=1946 \pm 48 \mathrm{MeV}$ ), extracted from the $\phi$-distributions of the final state $\eta$-meson. Solid (black) curve: BnGa-PWA [43, 44], long-dashed (green) curve: Fix et al. [120, 121], short-dashed (red) curve: Döring et al. [122].
$\Delta(1700) 3 / 2^{-} \rightarrow \Delta \pi$, one preferred $D$-wave. Both solutions are shown in Fig. 24, bottom, as dashed and solid curves, respectively. The sensitivity of these observables to the two decay modes is clearly visible. Additionally, a prediction by Arenhövel et al. [123] is shown (red line), assuming $N(1520) 3 / 2^{-}$(and not $\Delta(1700) 3 / 2^{-}$) to be the dominant isobar to the reaction, and assuming $\Delta \pi$ to be the dominant decay mode of resonances. These assumptions are distinctively dismissed by the data.

The importance of $N(1520) 3 / 2^{-}$as an intermediate resonance in cascade decays of higher-lying resonances is demonstrated in Fig. 25. The Dalitz plot is shown for $1300<E_{\gamma}<1650 \mathrm{MeV}$ which covers the invariant mass range from 1820 to $2000 \mathrm{MeV}$. For this mass range, $I^{S}$ and $I^{c}$ have been extracted from the data for two different regions, I and II, of the Dalitz plot, as denoted by the lines. In the region where $\Delta(1232) 3 / 2^{+}$dominates the intermediate state, the $I^{s}$ and $I^{c}$ show little to no amplitude (see the upper row of the four subfigures in Fig. 25). In region II where the cascade $N^{*} \rightarrow N(1520) \pi^{0} \rightarrow p \pi^{0} \pi^{0}$ makes a significant contribution, $I^{s}$ and $I^{c}$ show a significant structure (see the lower row of the four subfigures in Fig. 25). The latter four distributions can be fitted with very simple assumptions. A resonance in one initial partial wave with defined $J^{P}$ is assumed to decay via
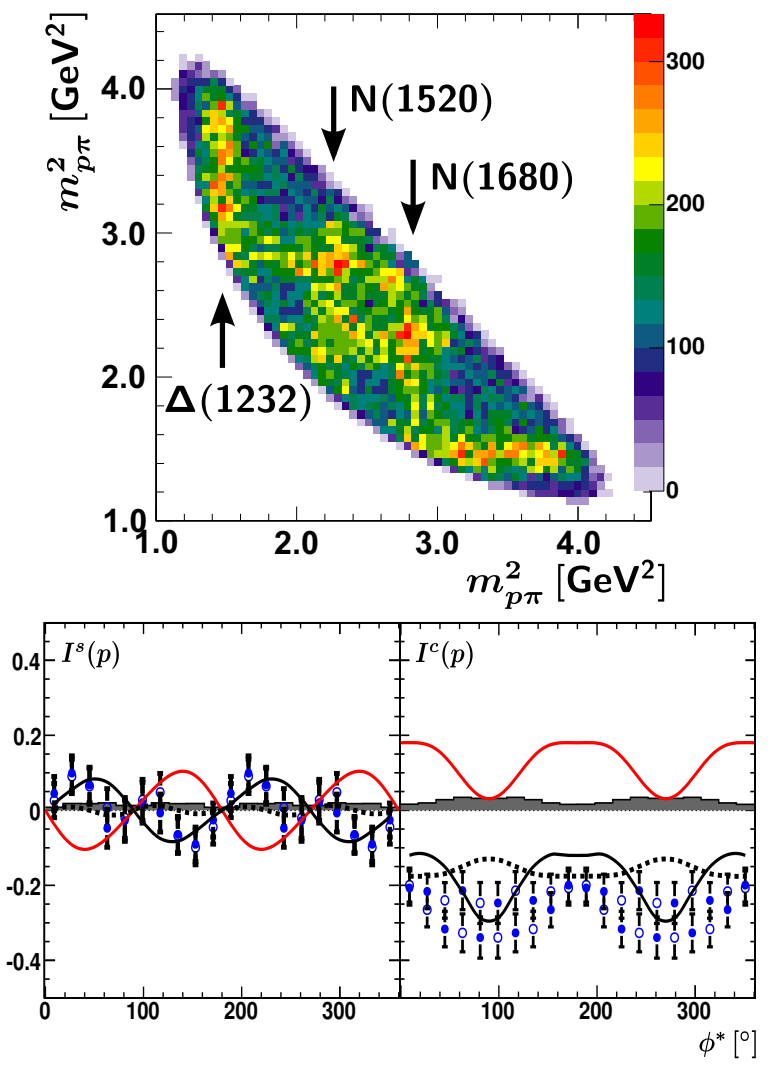

Figure 24. Top: Dalitz plot for the reaction $\gamma p \rightarrow p \pi^{0} \pi^{0}$, exhibiting a rich spectrum of intermediate resonance structures $\left(E_{\gamma}=2000\right.$ $2100 \mathrm{MeV})$. Bottom: Exemplary distributions for the observables $I^{s}$ and $I^{c}\left(E_{\gamma}=970-1200 \mathrm{MeV}\right)$, extracted from the $\phi$-distributions of the final state proton. Solid (black) curve: BnGa-PWA [53, 54] with $D$-wave dominance $\Delta(1700) 3 / 2^{-} \rightarrow \Delta \pi$, dashed: BnGa-PWA with $S$-wave dominance $\Delta(1700) 3 / 2^{-} \rightarrow \Delta \pi$, solid, (red): Arenhövel et al. [123]. 
a cascade $N^{*} \rightarrow N^{+}(1520) \pi^{0} \rightarrow p \pi^{0} \pi^{0}$. It turns out that fits with $J^{P}=1 / 2^{+}, 5 / 2^{+}$and $7 / 2^{+}$give a bad description while a fit with $J^{P}=3 / 2^{+}$yields a good description of the data. In the $\mathrm{W} \approx 1900 \mathrm{MeV}$ region, the cascade $N^{*} \rightarrow N(1520) \pi^{0} \rightarrow p \pi^{0} \pi^{0}$ is dominated by the $J^{P}=3 / 2^{+}$ partial wave (see Fig. 6 in the contribution of project A.2 to these proceedings and [56] for a detailed discussion).

These observations made directly from the data are confirmed by the BnGa multi-channel partial wave analysis (project A.2) which includes a large number of pion and photo-induced reactions in addition to the data presented here. The result of a mass scan in which a single Breit-Wigner amplitude with $J^{P}=3 / 2^{+}$is added above $N(1720) 3 / 2^{+}$to the set of amplitudes is shown in Fig. 26. The mass of the added $J^{P}=3 / 2^{+}$-state is fixed while all other parameters are fitted freely. The $\chi^{2}$-difference, normalized to the minimal $\chi^{2}$ of the fit, shows a clear minimum in the respective mass range. The same is true if the $\chi^{2}$-change only due to the $\gamma p \rightarrow p \pi^{0} \pi^{0}$-reaction is looked at.

The BnGa-PWA provides additionally indications for the existence of a second resonance around $1900 \mathrm{MeV}$ in the $3 / 2^{+}$wave beyond the known $N(1900) 3 / 2^{+}$[56] even though the fit to the data with $N(1900) 3 / 2^{+}$only is also acceptable.
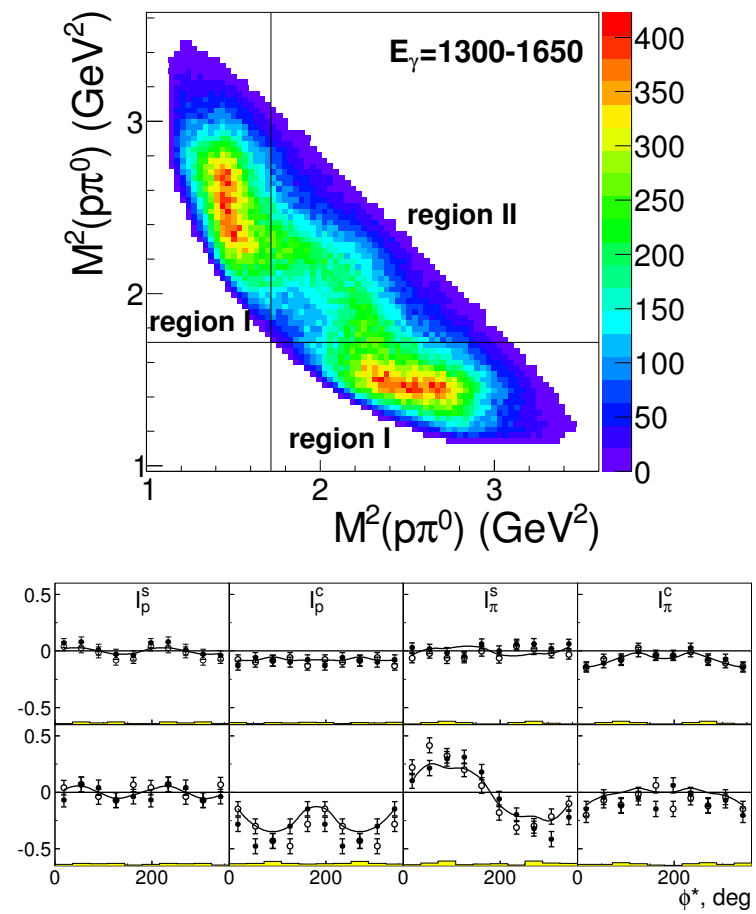

Figure 25. Top: Kinematic regions in the Dalitz plot used to study the reaction dynamics $\left(E_{\gamma}=1300-1650 \mathrm{MeV}\right)$. The phase space is divided into two regions I and II. In region II the $\Delta(1232)$ contribution is suppressed and the $N(1520) 3 / 2^{-}$contribution enhanced. Bottom: $I_{p}^{s}, I_{p}^{c}, I_{\pi}^{s}$, and $I_{\pi}^{c}$ as functions of $\phi^{*}$ for $1300<E_{\gamma}<1650 \mathrm{MeV}$. The subfigures on the first (second) row show the distributions for events in region I (II) of the Dalitz plot. Dots: $I^{s}, I^{c}$; open circles: derived from symmetry; yellow band: syst. uncertainties. The solid curve represents a two-parameter fit assuming $J^{P}=3 / 2^{+}$as initial partial wave (Figures taken from [56]).

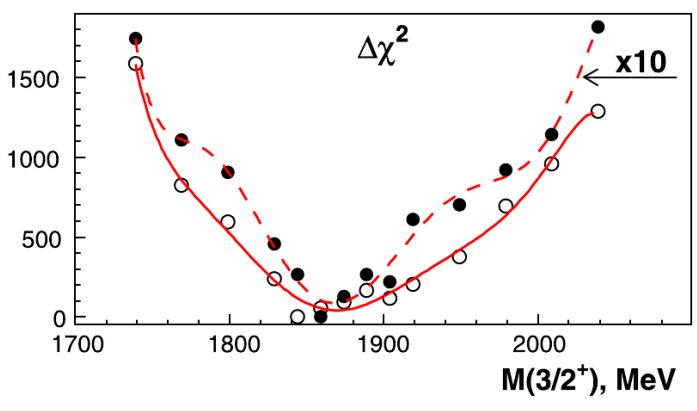

Figure 26. Mass scan for a resonance with $J^{P}=3 / 2^{+}$. Shown is the $\chi^{2}$-change of the BnGa fit as a function of the imposed mass. In the mass scan, a Breit-Wigner amplitude with $J^{P}=3 / 2^{+}$is added above $N(1720) 3 / 2^{+}$; its mass is fixed while all other parameters are fitted freely. The curves are included to guide the eye. The open circles give the total change in $\chi^{2}$, normalized to the minimal $\chi^{2}$. The black circles represent the $\chi^{2}$-contribution from $p \pi^{0} \pi^{0}$; it is multiplied by a factor 10 for better visibility. The $\gamma p \rightarrow p \pi^{0} \pi^{0}$ data are fitted by an event-based likelihood fit; the likelihood converted into a pseudo- $\chi^{2}$ by $\Delta(\ln L)=2 \Delta \chi^{2}$. (Figure taken from [57]).

The partial wave analysis (within the Bonn-Gatchina approach) of the multi-meson data yields a large number of branching ratios of resonances into $\Delta \pi, \Delta \eta$, $N(\pi \pi)_{S}, N(1440) 1 / 2^{+}, N(1520) 3 / 2^{-}, N(1535) 1 / 2^{-}$, and $N(1680) 5 / 2^{+}$. Many of these branching ratios have been determined for the first time. Decays of $\Delta(1910) 1 / 2^{+}$, $\Delta(1920) 3 / 2^{+}, \Delta(1905) 5 / 2^{+}, \Delta(1950) 7 / 2^{+}$into $\Delta(1232) \pi$ and of the corresponding spin-parity series in the nucleon sector, $N(1880) 1 / 2^{+}, N(1900) 3 / 2^{+}, N(2000) 5 / 2^{+}$, and $N(1990) 7 / 2^{+}$are observed. For the nucleon resonances, these decay modes were reported for the first time. Further new decay modes proceed via $N(1520) 3 / 2^{-} \pi$, $N(1535) 3 / 2^{-} \pi, N(1680) 5 / 2^{+} \pi$, and $N \sigma$. The latter decay modes are observed in the decay of $N^{*}$ resonances, in $\Delta^{*}$ decays, however, at most weakly. In $[55,56]$ it is argued that these decay modes provide evidence for a 3-quark nature of $N^{*}$ resonances rather than a quark-diquark structure (for further explanations see also the contribution of project A. 2 to these proceedings).

\section{Photoproduction off neutrons}

\subsection{Study of a narrow peak in the $n \eta$ mass distribution}

A narrow dip-bump structure at $1.67 \mathrm{GeV}$ was observed in photoproduction of $\eta$ mesons off neutrons [124]. It is discussed in more detail in the contribution off project A.2 "Partial Wave Analysis" to these proceedings. The peaking structure was observed in the $n \eta$ invariant mass distribution but not seen in the cross section on the proton. The structure was suggested to signal the existence of a relatively narrow $(M \approx 1.68 \mathrm{GeV}, \Gamma \leq 30 \mathrm{MeV})$ baryon state.

The CBELSA/TAPS collaboration studied the reaction $\gamma d \rightarrow \eta n p_{\text {spectator }}$ and confirmed the structure [125] in the $n \eta$ invariant mass while no similar structure was observed in the $n \eta^{\prime}$ mass distribution [126]. The structure became even more pronounced when the recoiling neutron was detected and effects from Fermi smearing were avoided [127]. In 2012, the structure was listed in the RPP 
as one-star resonance. At Mainz, the structure was studied with high statistics and again confirmed [128, 129]. The peak is discussed controversially in the literature, and different interpretations were given [130-136]. So far, the most recent and most precise data $[128,129]$ were only fitted in [136]. The latter fit gave a nearly perfect description of the data assuming that the structure emerges from the interference of $N(1535) 1 / 2^{-}$and $N(1650) 1 / 2^{-}$within the $1 / 2^{-}$-partial wave. The fit deteriorated when in the fit a resonance was imposed with properties as suggested in the experimental studies. In 2016, the PDG decided to remove $N(1685)$ from the listings.

\subsection{Helicity amplitudes off neutrons}

Data on photoproduction off neutrons provide for the respective $\gamma n$-helicity amplitudes. To obtain sufficient information, all existing data on $\gamma n$ were included in the BnGa partial wave analysis [135]. The fit was further constrained by the data photoproduction off protons. Recently, CLAS reported new and very precise data on $\gamma n \rightarrow K^{0} \Lambda$; the new data allowed for a new determination of the helicity amplitudes for photoproduction of neutrons [137].

\section{Recent results}

In this section we present results which are based on data taken during the SFB which are not yet published or submitted for publication and which partly are still preliminary.

\section{$7.1 \gamma p \rightarrow p \pi^{0} \pi^{0}$ : The polarization observables $\boldsymbol{T}, \boldsymbol{P}, \mathrm{H}$}

The data taken with transversely polarized target (see Section 4.4 and 4.6) were also analyzed to investigate the reaction $\gamma p \rightarrow \pi^{0} \pi^{0} p \rightarrow 4 \gamma p$ [139]. The four photons were combined pair-wise to form the pions. The resulting invariant mass spectrum is shown in Fig. 27 where the mass of one photon pair is plotted against the other. Peaks due to the double pion final state as well as the $\pi^{0} \eta$ final state are clearly visible. The shown spectrum still contains combinatorical background which can be eliminated nearly completely by employing a kinematic fit using the masses of the final state particles as constraints. The background contamination in the final data set is in the order of $1.5 \%$. The dilution factor has been determined again by analyzing the $\mathrm{C}$-foam-target data as described in Section 3.5 .

Taking the full 3-body-kinematics of the $p \pi^{0} \pi^{0}$ final state into account additional polarization observables, not present for two-body final states, arise. Considering only linearly polarized photons and transversely polarized tar-

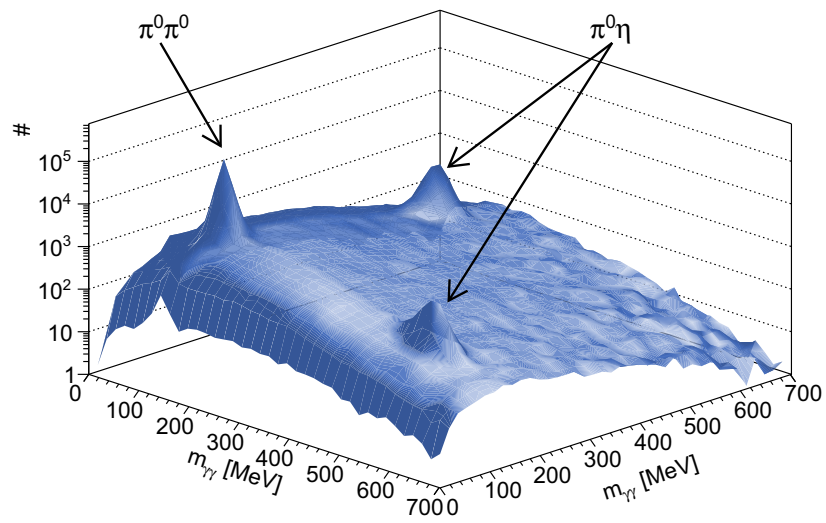

Figure 27. Invariant mass of one photon pair plottet versus the invariant mass of the other. Combinatorical background (which can be eliminated) is still included here.

get protons, the differential cross section is given by [63]

$$
\begin{aligned}
\frac{\mathrm{d} \sigma}{\mathrm{d} \Omega}=\frac{\mathrm{d} \sigma_{0}}{\mathrm{~d} \Omega} & \cdot\left\{\left[1+\Lambda_{x} P_{x}+\Lambda_{y} P_{y}\right]\right. \\
& +\delta_{\ell} \cdot \sin (2 \phi) \cdot\left[I^{s}+\Lambda_{x} P_{x}^{s}+\Lambda_{y} P_{y}^{s}\right] \\
& \left.+\delta_{\ell} \cdot \cos (2 \phi) \cdot\left[I^{c}+\Lambda_{x} P_{x}^{c}+\Lambda_{y} P_{y}^{c}\right]\right\}
\end{aligned}
$$

with the degree of linear polarization $\delta_{\ell}$ and the target polarization degree parallel (perpendicular) to the reaction plane $\Lambda_{x}\left(\Lambda_{y}\right)$. After integration over $\phi^{*}$, the polarization observables $P_{y}, P_{y}^{c}$ and $P_{x}^{s}$ correspond to the observables $T, P$, and $H$ in photoproduction of single pseudoscalar mesons (Fig. 22, left).

As an example, the target asymmetry $P_{y}$ is shown in Fig. 28 as a function of the angle $\phi^{*}$. Here only every second energy bin is shown. The PWA predictions from the BnGa group show significant discrepancies to the data: The data shows a much more pronounced $\phi^{*}$-dependence than the BnGa'2014-predictions. Results for the double polarization observables $P$ and $H$ are shown in Fig. 29 as functions of the invariant mass of the two pions. The PWA predictions completely fail to describe the data indicating again that double pion photoproduction is not yet fully understood.

Unlike the single meson photoproduction where there are only two kinematic variables (e.g. $E_{\gamma}, \cos \vartheta$ ), a three body final state like $p \pi^{0} \pi^{0}$ has a five-dimensional phase space. For a complete experiment the polarization observables need to be determined at every (5-dimensional) kinematic point. Therefore, it is important to determine the observables multi-dimensional. Fig. 30 shows the target asymmetry $P_{y}$ for one energy bin simultaneously binned in $\cos \vartheta, m_{p \pi^{0}}$ and $\phi^{*}$. The observable clearly shows quite a different behavior in different kinematic regions. Obviously the integrated target asymmetry shown in Fig. 28 carries only a part of the full information. In Fig. 28, all data shown in Fig. 30 are contained in the third energy bin of Fig. 28 (and the two bins adjacent in energy which are not shown). The data ist presently included in the PWA 


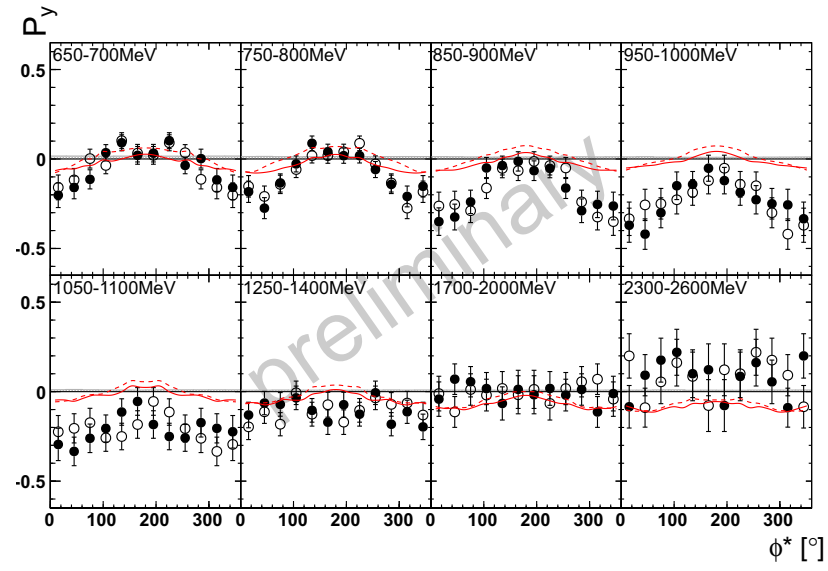

Figure 28. Target asymmetry $P_{y}$ as a function of $\phi^{*}$ (black dots). The open symbols are calculated from the symmetry property $P_{y}\left(2 \pi-\phi^{*}\right)=P_{y}\left(\phi^{*}\right)$. Red solid line: predictions from BnGa'2014-02, red dashed line: BnGa'2014-01.

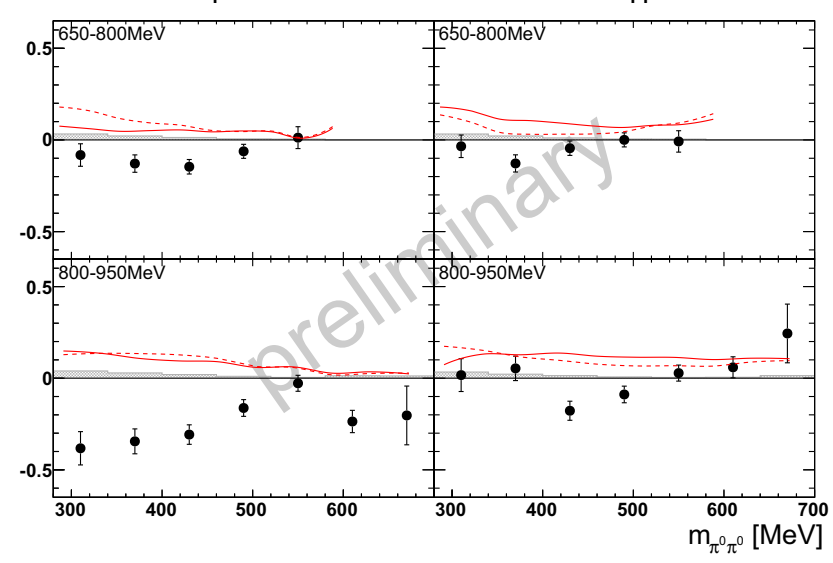

Figure 29. Double polarization observables $P$ and $H$ as a function of the double pion invariant mass. Predictions from BnGa'2014-02 (red solid line) and BnGa'2014-01 (red dashed) fail to describe the data.

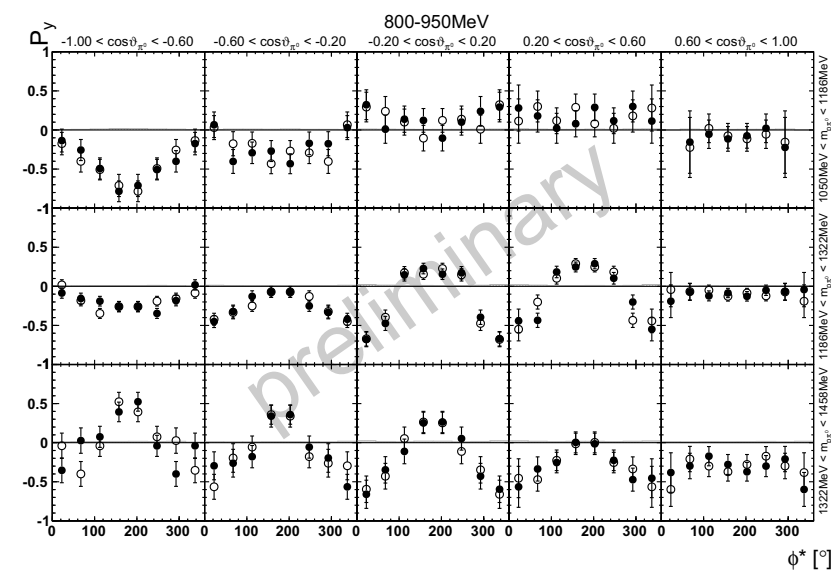

Figure 30. Four-dimensional determination of the target asymmetry $P_{y}$ : Shown is the energy bin $800-950 \mathrm{MeV}$, within one row only $\cos \vartheta$ is varied, within one column only $m_{p \pi^{0}}$ and $\phi^{*}$ is on the $x$-axis of the individual histograms. The open symbols are calculated from the symmetry property $P_{y}\left(2 \pi-\phi^{*}\right)=P_{y}\left(\phi^{*}\right)$. and one can expect that it will help to constrain the $p 2 \pi^{0}$ decays of $N^{*}$ and $\Delta^{*}$-resonances more precisely.

\section{$7.2 \gamma p \rightarrow p \pi^{0} \pi^{0}:$ The beam asymmetry $\Sigma$ at high energies}

In the fall of 2013, a large-statistics data set with a linearly polarized photon beam impinging on an unpolarized liquid hydrogen $\left(\mathrm{LH}_{2}\right)$ target was taken. The coherent edge was placed at remarkably high beam energies of $1750 \mathrm{MeV}$ resp. $1850 \mathrm{MeV}$. This allows for studies of the beam asymmetry $\Sigma$ (or of $I^{c}$ and $I^{s}$ in the case of two pseudoscalar mesons in the final state) without the problems stemming from the inherent carbon dilution of the polarized target. The analysis of the reaction $\gamma p \rightarrow \pi^{0} \pi^{0} p$ is currently ongoing [138]. Due to the five-dimensional reaction phase space, it will especially benefit from the large amount of data that has been recorded. Within a preliminary reaction selection, $3.6 \times 10^{6} 2 \pi^{0}$ events were reconstructed.

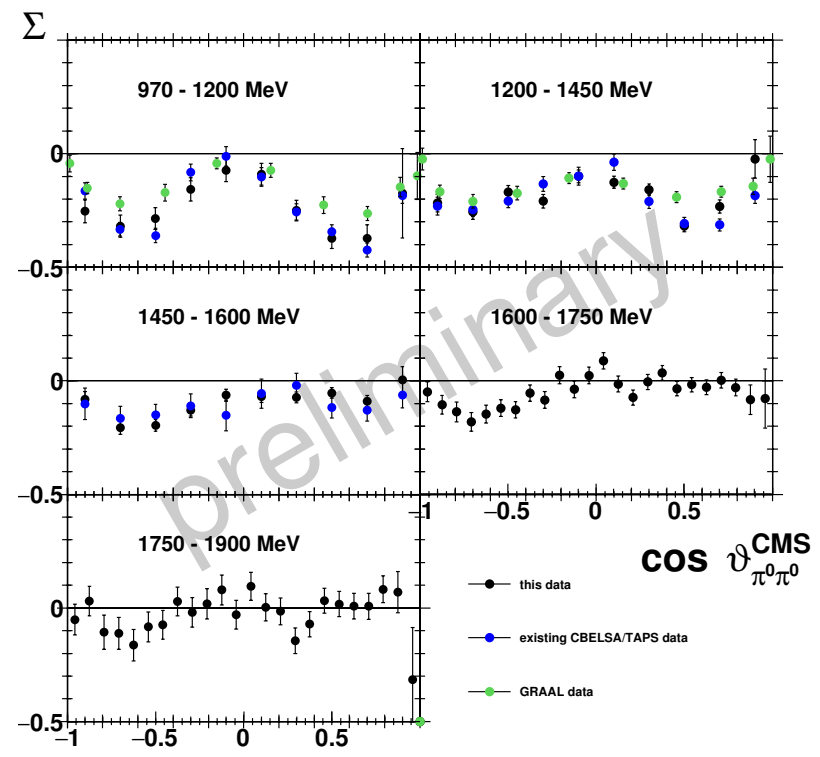

Figure 31. Preliminary CBELSA/TAPS-results (black) on the polarization observable $\Sigma$ in the quasi-two-body approach, the error bars denote the statistical error only. For the first three energy bins, the angular binning is chosen to be compatible with the existing results (GRAAL [51]: green, published lower energy CBELSA/TAPS data [56, 57]: blue). Higher beam energies are shown with higher resolution of the polar angle of the mesonsystem.

A first view on the data is shown in Fig. 31. For lower beam energies, the data can be compared to existing results from the GRAAL [51] and the CBELSA/TAPS collaboration $[56,57]$. Compared with the GRAAL data, the beam asymmetry is found with a slightly larger amplitude, but in good agreement with the CBELSA/TAPS data. Possibly, the differences are caused by a difference in the acceptance coverage of the two detector systems. Note that at higher energies in the region of higher polarization, $\Sigma$ is shown with a finer binning. 


\section{$7.3 \gamma p \rightarrow p \pi^{0} \pi^{0}:$ Preliminary results on the double polarization observables $E$}

The double polarization observable $E$ is currently also extracted for two neutral pions in the final state [138]. Compared to single meson photoproduction, the required polarization settings (circularly polarized beam with polarization $\delta_{\odot}$ and longitudinally polarized target with polarization $\Lambda_{z}$ ) give rise to further polarization observables. Following [63] the relevant part of the cross-section reads:

$$
\frac{\mathrm{d} \sigma}{\mathrm{d} \Omega}=\frac{\mathrm{d} \sigma_{0}}{\mathrm{~d} \Omega} \cdot\left[1+\delta_{\odot} I^{\odot}+\Lambda_{z} P_{z}+\Lambda_{z} \delta_{\odot} P_{z}^{\odot}\right],
$$

where $P_{z}^{\odot}$ corresponds to $-E$ in single meson photoproduction if integrated over $\phi^{*}$.

Changing the sign of the beam and the target polarization, the data set can be divided into four groups; the polarization observables are then accessible as pairwise asymmetries between the subsets. However, the single polarization observables are still under investigation $\left(P_{z}\right)$ or should be measured with a liquid-hydrogen target $\left(I^{\odot}\right)$ to avoid the data being diluted with reactions off bound protons. In the following, preliminary results for the polarization observable $E$ will be shown.

Events in the detector with one charged and four neutral hits (considered as the decay photons of the two pions) are further selected by means of orthogonal cuts. Cut boundaries are fine-tuned to match the experimental conditions at different kinematic regions and account for the target field direction, as the longitudinal magnetic holding field influences the trajectory of the proton in azimuthal direction. So far, the data still includes combinatorial background. The carbon contribution still remaining after the kinematic cuts is eliminated by subtracting the carbon yield, measured separately and scaled correctly, from each of the subsets mentioned above.

Overall, approximately $5 \times 10^{5}$ events in the dataset fulfill the cut conditions. Thus, the five-dimensional reaction phase space is explored by various (generally 2dimensional) projections. Figure 32 shows the helicity asymmetry as a function of the beam energy and the polar angle of the vector sum of the meson system. Instead of the proton, one pion can be considered as the recoiling particle, which is used for the projections of figure 33. Compared to the fully-predictive partial wave analysis solutions, discrepancies are still occurent, most visible at medium or highest beam energies. Within a three-body approach, the resulting observable can be tested to be invariant under parity conservation, which translates into the constraint $E\left(\phi^{*}\right)=E\left(2 \pi-\phi^{*}\right)$ and appears to be in good agreement with the data. After the data analysis is finalised the data will be included in the BnGa-PWA.

\section{Summary and Outlook}

One of the most important topics in baryon spectroscopy is to identify the relevant degrees of freedom and the effective forces which determine the spectrum and properties of baryon resonances. Here significant progress has been

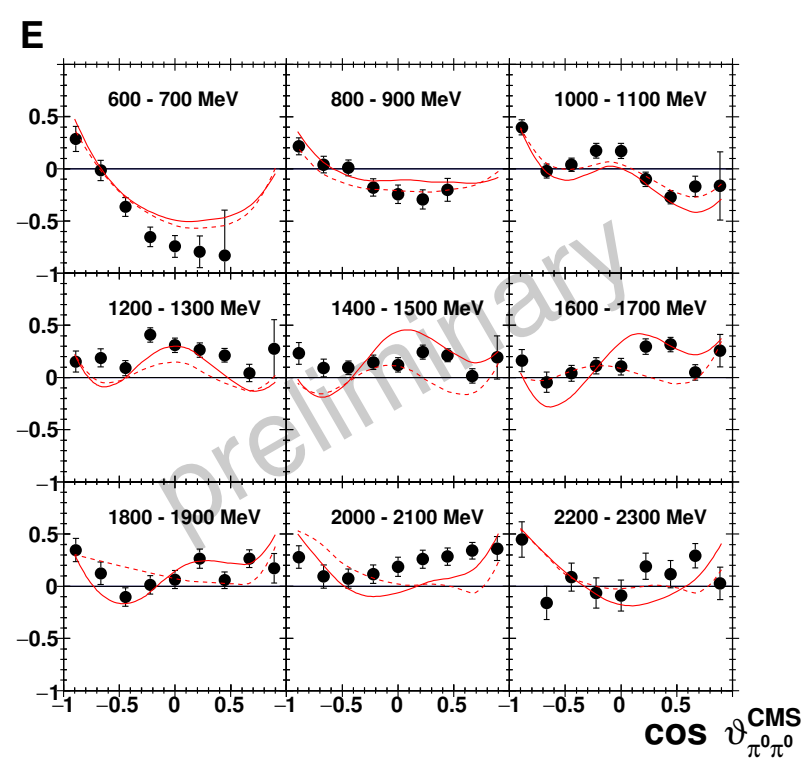

Figure 32. Preliminary results on the polarization observable $E$ in the two-body approach. The error bars denote the statistical error. Only every second energy bin is shown. Results are shown along with predictions of the Bonn-Gatchina partial wave analysis group (- BnGa 2014-02 --- BnGa 2014-01)
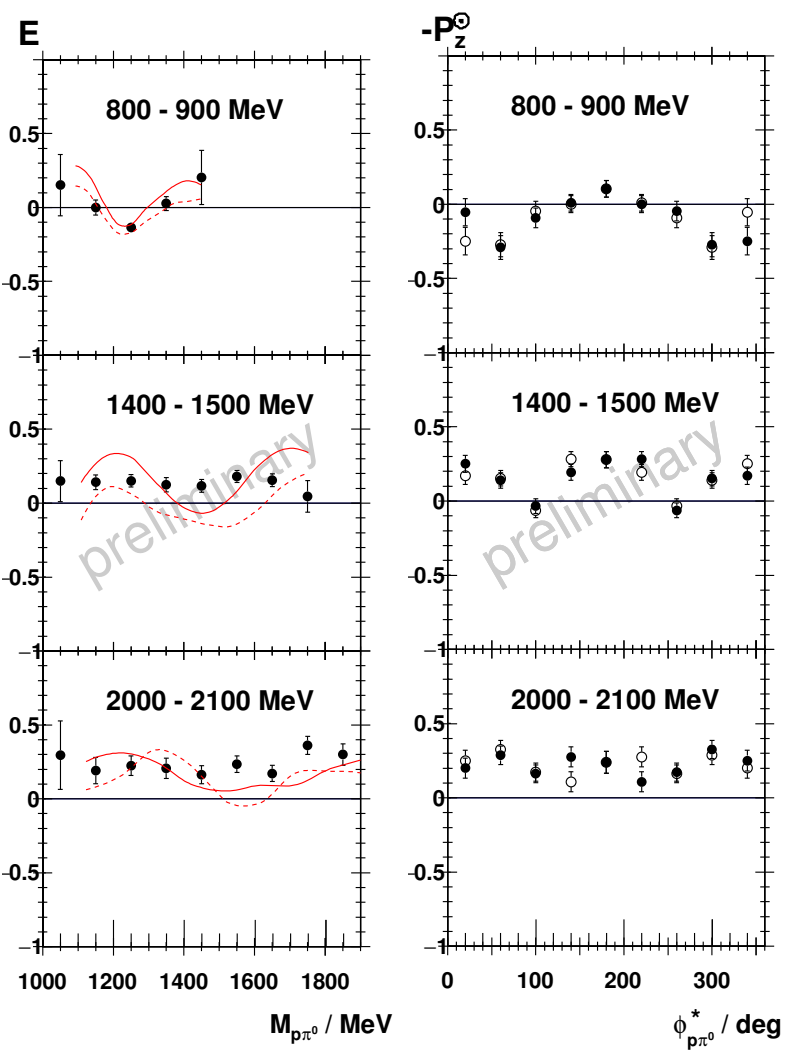

Figure 33. Preliminary results on the polarization observable $E$ in two-body and three-body (right) approach, the error bars denote the statistical error. Only selected energy bins are shown. On the left hand side, the data are compared to the predictions of the Bonn-Gatchina partial wave analysis group (- BnGa 201402, --- BnGa 2014-01). Within a three-body approach, the resulting observable can be tested to be invariant under parity conservation, which translates into the constraint $E\left(\phi^{*}\right)=E\left(2 \pi-\phi^{*}\right)$. The open symbols show this symmetrization of the data. 

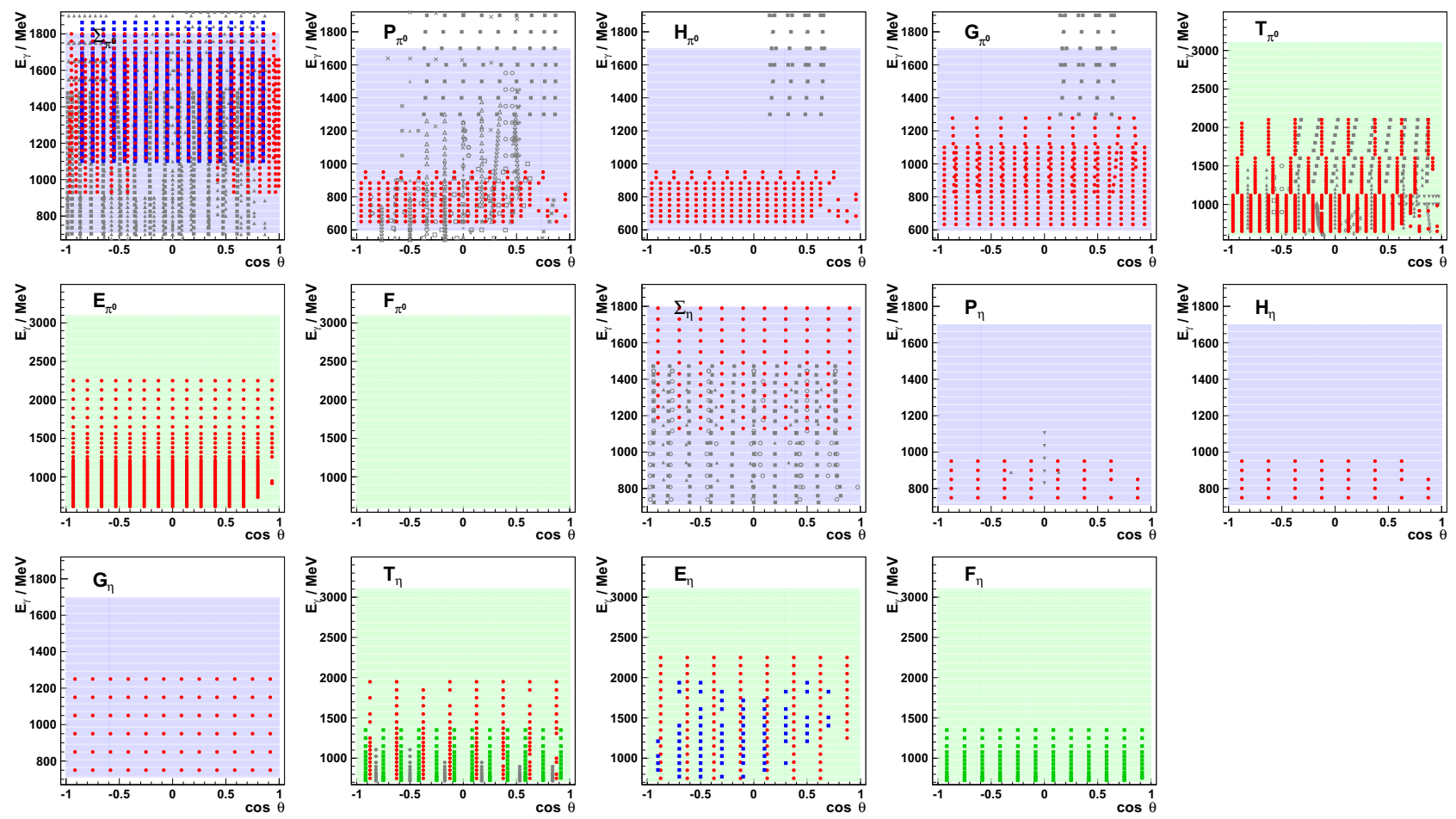

Figure 34. Gray: Existing data (SAID-data base), red: new published data from the CBELSA/TAPS-experiment, blue: new CLASdata, green: new MAMI data. Green (blue area): energy regime accessible at ELSA with circularly (linearly) polarised photons. Of course the target polarization $T$ can be determined independently from the beam-polarization.

made. Based on the new high statistics unpolarized and polarized data taken at ELSA and elsewhere, new baryon resonances could be identified and known or less well known states could be confirmed or have in part been reestablished. The number of the now observed resonances excludes e.g. quasi-stable diquarks as part of the dynamics of excited baryons. In addition, based on the new photoproduction data, properties of the baryon resonances such as their partial decays widths in different final states could be determined (see contribution of the project A.2 "Partial Wave Analysis" to these proceedings).

Of course not all of the resonances found in the data are considered to be established ( $\geq 3$-star) resonances yet. Further and higher statistics data is needed. Higher statistics is especially needed at higher energies and for channels with lower cross section. The need for the measurement of additional polarization observables relates to areas in which so far no, or only very restriced data has been taken. Fig. 34 shows for two reactions $\gamma p \rightarrow p \pi^{0}$ and $\gamma p \rightarrow p \eta$ the polarization observables which have been investigated. Data in the so far not covered regions (Figs. 34), which include especially the regime of higher energies/masses are clearly of large interest. Respective measurements which provide of course not only access to $\pi^{0}$ - and $\eta$-photoproduction but also to additional single and multi-meson final states are planned at ELSA. Here it needs to be stressed, that especially also reactions with more than a single pseudoscalar meson in the final state can be expected to provide new and interesting results on the presently least understood higher mass baryon spec- trum. These measurements will extend our knowledge of the baryon spectrum into the regime of higher masses.

Another interesting and so far not well investigated area is photoproduction of the neutron: baryon resonances may strongly couple to the neutron but they may only weakly or not at all couple to the proton. Therefore resonances, maybe even resonances of specific symmetries or nature, may escape detection if only proton data is analyzed. This bias can be overcome if also photoproduction data off the neutron, which is presently still scarce, is taken and investigated. Another not well investigated degree of freedom in the experiments is the isospin dependence. The electromagnetic transition operator can be split into an isovector and an isoscalar part, giving rise to three independent matrix elements [140]: $A^{I S}=\left\langle\frac{1}{2}, \pm \frac{1}{2}|\hat{S}| \frac{1}{2}, \pm \frac{1}{2}\right\rangle, \quad \mp A^{I V}=\left\langle\frac{1}{2}, \pm \frac{1}{2}|\hat{V}| \frac{1}{2}, \pm \frac{1}{2}\right\rangle$, $A^{V 3}=\left\langle\frac{3}{2}, \pm \frac{1}{2}|\hat{V}| \frac{1}{2}, \pm \frac{1}{2}\right\rangle$, using the notation $\left\langle I_{f}, I_{f 3}|\hat{O}| I_{i}, I_{i 3}\right\rangle$. Photoproduction of isovector mesons, such as the pions, involve all three matrix elements, while in the case of isoscalar meson photoproduction only two, $A^{I S}$ and $A^{I V}$, contribute. To study the isospin structure of the amplitudes, photoproduction experiments on the neutron are unavoidable. This becomes directly obvious in the isoscalar meson production. It is hence important to study photoproduction off neutrons with resonable precision compared to what was achieved for protons. Respective measurements will be performed at ELSA.

The work reported here would not have been possible without the enduring enthusiasm of many bachelor, master, $\mathrm{PhD}$ students, and post-docs as well as the continuous technical support 
from staff members of the participating Universities. The support of the ELSA staff members and the polarized target group is gratefully recognized. The results discussed here have been obtained within the CBELSA/TAPS collaboration. At the beginning of the SFB/TR16, Christian Weinheimer was the designated A.1 project leader but accepted a professorship at the University of Münster shortly after the start of the SFB/TR16. Rainer Novotny was project leader of A.1 during the first funding period focussing on the hardware developments. With the second funding period project A.1 was split into the project presented here and the instrumentation project D.3. The instrumentation results including the ones obtained during the first funding period have been summarized within project D.3. We would like to thank E. Klempt, F. Afzal, J. Hartmann, P. Mahlberg, H. van Pee, T. Seifen, A. Thiel, and Y. Wunderlich for contributions to this report. We appreciate the support of the Schweizerischer Nationalfonds, the US National Science Foundation, and the Russian Foundation for Basic Research, in particular the generous support from the Deutsche Forschungsgemeinschaft (SFB/TR16).

\section{References}

[1] V. E. Barnes et al., "Observation of a Hyperon with Strangeness -3," Phys. Rev. Lett. 12, 204 (1964).

[2] M. Gell-Mann, "A Schematic Model Of Baryons And Mesons,” Phys. Lett. 8, 214 (1964).

[3] G. Zweig, "An SU(3) Model For Strong Interaction Symmetry And Its Breaking.," published in 'Developments in the Quark Theory of Hadrons'. Volume 1. Edited by D. Lichtenberg and S. Rosen. Nonantum, Mass., Hadronic Press, 1980. pp. 22-101.

[4] O. W. Greenberg, "Spin and Unitary Spin Independence in a Paraquark Model of Baryons and Mesons," Phys. Rev. Lett. 13, 598 (1964).

[5] M. Y. Han and Y. Nambu, "Three Triplet Model with Double SU(3) Symmetry," Phys. Rev. 139, B1006 (1965).

[6] S. Weinberg, "Nonabelian Gauge Theories Of The Strong Interactions," Phys. Rev. Lett. 31, 494 (1973).

[7] H. Fritzsch, M. Gell-Mann, H. Leutwyler, "Advantages of the Color Octet Gluon Picture," Phys. Lett. B47, 365 (1973).

[8] K. G. Wilson, "Confinement of Quarks," Phys. Rev. D10, 2445 (1974).

[9] S. Coleman and D. J. Gross, "Price of Asymptotic Freedom,” Phys. Rev. Lett. 31, 851 (1973).

[10] D. J. Gross and F. Wilczek, "Ultraviolet Behavior of Nonabelian Gauge Theories," Phys. Rev. Lett. 30, 1343 (1973).

[11] H. D. Politzer, "Reliable Perturbative Results for Strong Interactions?,” Phys. Rev. Lett. 30, 1346 (1973).

[12] N. Isgur and G. Karl, "Hyperfine Interactions in Negative Parity Baryons," Phys. Lett. B 72, 109 (1977).

[13] N. Isgur and G. Karl, "P Wave Baryons in the Quark Model,” Phys. Rev. D 18, 4187 (1978).

[14] J. Beringer et al. (Particle Data Group), Phys. Rev. D86, 010001 (2012).
[15] S. Bethke, G. Dissertori and G. P. Salam, "Quantum Chromo Dynamics", in: [16]

[16] K. A. Olive, "Review of Particle Physics," Chin. Phys. C 38, 090001 (2014).

[17] J. J. Aubert et al., "Experimental Observation of a Heavy Particle J,” Phys. Rev. Lett. 33, 1404 (1974).

[18] J. E. Augustin et al., "Discovery of a Narrow Resonance in $e^{+} e^{-}$Annihilation," Phys. Rev. Lett. 33, 1406 (1974).

[19] D. Robson, "A Basic Guide for the Glueball Spotter," Nucl. Phys. B 130, 328 (1977).

[20] M. S. Chanowitz and S. R. Sharpe, "Hybrids: Mixed States of Quarks and Gluons," Nucl. Phys. B 222, 211 (1983), Erratum: [Nucl. Phys. B 228, 588 (1983)].

[21] G. Arnison et al. [UA1 Collaboration], "Experimental Observation of Isolated Large Transverse Energy Electrons with Associated Missing Energy at $s^{1 / 2}=$ 540 GeV," Phys. Lett. B 122, 103 (1983).

[22] D. Decamp et al. [ALEPH Collaboration], "Determination of the Number of Light Neutrino Species," Phys. Lett. B 231, 519 (1989).

[23] G. Aad et al. [ATLAS Collaboration], "Observation of a New Particle in the Search for the Standard Model Higgs Boson with the ATLAS Detector at the LHC," Phys. Lett. B 716, 1 (2012).

[24] A. Freitas, "Understanding Electroweak Physics in the Standard Model and Beyond," Nucl. Part. Phys. Proc. 273-275, 21 (2016).

[25] S. Weinberg, "Phenomenological Lagrangians," Physica A 96, 327 (1979).

[26] M. Gell-Mann, R.J. Oakes and B. Renner, "Behavior of Current Divergences under SU(3) x SU(3)," Phys. Rev. 175, 2195 (1968).

[27] Y. Nambu, "Quasi-particles and Gauge Invariance in the Theory of Superconductivity," Phys. Rev. 117, 648 (1960).

[28] J. Goldstone, "Field Theories With Superconductor Solutions," Nuovo Cim. 19, 154 (1961).

[29] J. Gasser and H. Leutwyler, "Chiral Perturbation Theory to One Loop," Annals Phys. 158, 142 (1984).

[30] V. Bernard and U.-G. Meißner, "Chiral Perturbation Theory,” Ann. Rev. Nucl. Part. Sci. 57, 33 (2007).

[31] L. Montanet et al. [Particle Data Group Collaboration], "Review of Particle Properties. Particle Data Group,” Phys. Rev. D 50, 1173 (1994).

[32] G. Höhler, F. Kaiser, R. Koch and E. Pietarinen, "Handbook of Pion Nucleon Scattering", Phys. Daten 12N1, 1 (1979).

[33] R. E. Cutkosky, C. P. Forsyth, J. B. Babcock, R. L. Kelly and R. E. Hendrick, "Pion - Nucleon Partial Wave Analysis", 4th Int. Conf. on Baryon Resonances, Toronto, Canada, Jul 14-16, 1980. Published in Baryon 1980:19 (QCD161:C45:1980).

[34] R. A. Arndt, W. J. Briscoe, I. I. Strakovsky and R. L. Workman, "Extended Partial-wave Analysis of $\pi N$ Scattering Data”, Phys. Rev. C 74, 045205 (2006).

[35] I. G. Alekseev et al., "Influence of Spin-rotation Measurements on Partial-wave Analyses of Elastic Pion 
Nucleon Scattering," Phys. Rev. C 55, 2049 (1997).

[36] I. G. Alekseev et al., "Measurements of Spin Rotation Parameter A in Pion Proton Elastic Scattering at $1.62 \mathrm{GeV} / \mathrm{c}$," Phys. Lett. B 485, 32 (2000).

[37] I. G. Alekseev et al., "Measurement of the Spin Rotation parameter A in the Elastic Pion Proton Scattering at $1.43 \mathrm{GeV} / \mathrm{c}$," Eur. Phys. J. C 45, 383 (2006).

[38] I. G. Alekseev et al., "Backward Asymmetry Measurements in the Elastic Pion-proton Scattering at Resonance Energies,” Eur. Phys. J. A 39, 163 (2009).

[39] A. V. Anisovich, E. Klempt, V. A. Nikonov, A. V. Sarantsev and U. Thoma, "Nucleon Resonances in the Fourth Resonance Region," Eur. Phys. J. A 47, 153 (2011).

[40] A. V. Anisovich, E. Klempt, V. A. Nikonov, A. V. Sarantsev, H. Schmieden and U. Thoma, "Evidence for a Negative-parity Spin-doublet of Nucleon Resonances at $1.88 \mathrm{GeV}$," Phys. Lett. B 711, 162 (2012).

[41] A. V. Anisovich, E. Klempt, V. A. Nikonov, A. V. Sarantsev and U. Thoma, "Evidence for a Spinquartet of Nucleon Resonances at $2 \mathrm{GeV}$," Phys. Lett. B 711, 167 (2012).

[42] A. V. Anisovich, R. Beck, E. Klempt, V. A. Nikonov, A. V. Sarantsev and U. Thoma, "Properties of Baryon Resonances from a Multichannel Partial Wave Analysis," Eur. Phys. J. A 48, 15 (2012).

[43] I. Horn et al. [CB-ELSA Collaboration], "Evidence for a Parity Doublet $\Delta(1920) P 33$ and $\Delta(1940) D 33$ from $\gamma p \rightarrow p \pi^{0} \eta$,' Phys. Rev. Lett. 101, 202002 (2008).

[44] I. Horn et al. [CB-ELSA Collaboration], "Study of the Reaction $\gamma p \rightarrow p \pi^{0} \eta$," Eur. Phys. J. A 38, 173 (2008).

[45] J. Ajaka et al., "Simultaneous Photoproduction of $\eta$ and $\pi^{0}$ Mesons on the Proton," Phys. Rev. Lett. 100, 052003 (2008).

[46] V. L. Kashevarov et al. [Crystal Ball at MAMI and TAPS and A2 Collaborations], "Photoproduction of $\pi^{0} \eta$ on Protons and the $\Delta(1700) D_{33}$ Resonance," Eur. Phys. J. A 42, 141 (2009).

[47] E. Gutz et al. [CBELSA and TAPS Collaborations], "Photoproduction of Meson Pairs: First Measurement of the Polarization Observable $I^{s}$," Phys. Lett. B 687, 11 (2010).

[48] E. Gutz et al. [CBELSA/TAPS Collaboration], "High Statistics Study of the Reaction $\gamma p \rightarrow p \pi^{0} \eta$," Eur. Phys. J. A 50, 74 (2014).

[49] F. Zehr et al., "Photoproduction of $\pi^{0} \pi^{0}$ and $\pi^{0} \pi^{ \pm}$ Pairs off the Proton from Threshold to the Second Resonance Region,” Eur. Phys. J. A 48, 98 (2012).

[50] V. L. Kashevarov et al., "Study of the $\gamma p \rightarrow \pi^{0} \pi^{0} p$ Reaction with the Crystal Ball/TAPS at the Mainz Microton,” Phys. Rev. C 85, 064610 (2012).

[51] Y. Assafiri et al., "Double $\pi^{0}$ Photoproduction on the Proton at GRAAL," Phys. Rev. Lett. 90, 222001 (2003).

[52] A. V. Sarantsev et al. [CB-ELSA and A2-TAPS Collaborations], "New Results on the Roper Resonance and the $P_{11}$ Partial Wave", Phys. Lett. B 659, 94 (2008).
[53] U. Thoma, M. Fuchs et al. [CB-ELSA Collaboration], " $N^{*}$ and $\Delta^{*}$-decays into $N \pi^{0} \pi^{0}$ ", Phys. Lett. B 659, 87 (2008).

[54] A. V. Sarantsev et al., "New Results on the Roper Resonance and the $P_{11}$ Partial Wave," Phys. Lett. B 659, 94 (2008).

[55] A. Thiel et al. [CBELSA/TAPS Collaboration], "Three-body Nature of $N^{*}$ and $\Delta^{*}$ Resonances from Sequential Decay Chains," Phys. Rev. Lett. 114, 091803 (2015).

[56] V. Sokhoyan et al. [CBELSA/TAPS Collaboration], "Data on $I^{s}$ and $I^{c}$ in $\vec{\gamma} p \rightarrow p \pi^{0} \pi^{0}$ Reveal Cascade Decays of N(1900) via N(1520) $\pi$," Phys. Lett. B 746, 127 (2015).

[57] V. Sokhoyan et al. [CBELSA/TAPS Collaboration], "High-statistics Study of the Reaction $\gamma p \rightarrow p 2 \pi^{0}$," Eur. Phys. J. A 51, 95 (2015), Erratum: [Eur. Phys. J. A 51, 187 (2015)].

[58] K. A. Olive, "Review of Particle Physics," Chin. Phys. C 40, 100001 (2016).

[59] I. Denisenko et al., " $N *$ Decays to $N \omega$ from New Data on $\gamma p \rightarrow \omega p$," Phys. Lett. B 755, 97 (2016).

[60] W. T. Chiang and F. Tabakin, "Completeness Rules for Spin Observables in Pseudoscalar Meson Photoproduction," Phys. Rev. C 55, 2054 (1997).

[61] Y. Wunderlich, R. Beck and L. Tiator, "The complete-experiment Problem of Photoproduction of Pseudoscalar Mesons in a Truncated Partial-wave Analysis," Phys. Rev. C 89, 055203 (2014).

[62] A. V. Anisovich et al., "Energy-independent PWA of the Reaction $\gamma p \rightarrow K^{+} \Lambda$," Eur. Phys. J. A 50, 129 (2014).

[63] W. Roberts and T. Oed, "Polarization Observables for Two-pion Production off the Nucleon" Phys. Rev. C 71, 055201 (2005).

[64] W. Hillert, "The Bonn Electron Stretcher Accelerator ELSA: Past and Future," Eur. Phys. J. A 28S1, 139 (2006).

[65] H. Olsen, L. C. Maximon, "Photon and Electron Polarization in High-Energy Bremsstrahlung and Pair Production with Screening," Phys. Rev. 114, 887 (1959).

[66] W. Hillert, M. Gowin and B. Neff, "A new injector for polarized electrons at ELSA," Symposium on the Gerasimov-Drell-Hearn Sum Rule and the Nucleon Spin Structure in the Resonance Region (GDH 2000), 14-17 Jun 2000. Mainz, Germany, Proceedings. Edited by D. Drechsel and L. Tiator. Singapore, World Scientific, 2001.

[67] D. Elsner et al. [CBELSA and TAPS Collaborations], "Measurement of the beam asymmetry in $\eta$ photoproduction off the proton," Eur. Phys. J. A 33, 147 (2007).

[68] H. Dutz et al., "The New Bonn frozen spin target for experiments with real photons," Nucl. Instrum. Meth. A 340, 272 (1994).

[69] R. Koniuk, N. Isgur, "Where have all the Resonances gone? An Analysis of Baryon Couplings in a Quark Model with Chromodynamics”, Phys. Rev. Lett. 44, 
845 (1980).

[70] O. Bartholomy, V. Crede, H. van Pee et al. [CBELSA Collaboration], "Neutral Pion Photoproduction off Protons in the Energy Range $0.3 \mathrm{GeV}<E_{\gamma}<$ 3 GeV," Phys. Rev. Lett. 94, 012003 (2005).

[71] H. van Pee, O. Bartholomy et al. [CB-ELSA Collaboration], "Photoproduction of $\pi^{0}$-mesons off Protons from the $\Delta(1232)$ region to $E_{\gamma}=3 \mathrm{GeV}$," Eur. Phys. J. A 31, 61 (2007).

[72] V. Crede et al. [CBELSA/TAPS Collaboration], "Photoproduction of Neutral Pions off Protons", Phys. Rev. C 84, 055203 (2011).

[73] V. Crede, O. Bartholomy et al. [CB-ELSA Collaboration], "Photoproduction of $\eta$ Mesons off Protons for $0.75 \mathrm{GeV}<E_{\gamma}<3 \mathrm{GeV}$," Phys. Rev. Lett. 94, 012004 (2005).

[74] O. Bartholomy et al. [CB-ELSA Collaboration], "Photoproduction of $\eta$-mesons off Protons," Eur. Phys. J. A 33, 133 (2007).

[75] F. Renard et al. [GRAAL Collaboration], "Differential Cross-section Measurement of $\eta$ Photoproduction on the Proton from Threshold to $1100 \mathrm{MeV}$,' Phys. Lett. B 528, 215 (2002).

[76] M. Dugger et al. [CLAS Collaboration], "Eta Photoproduction on the Proton for Photon Energies from $0.75 \mathrm{GeV}$ to $1.95 \mathrm{GeV}$," Phys. Rev. Lett. 89, 222002 (2002), Erratum: [Phys. Rev. Lett. 89, 249904 (2002)].

[77] V. Crede et al. [CBELSA/TAPS Collaboration], "Photoproduction of $\eta$ and $\eta^{\prime}$ mesons off protons", Phys. Rev. C 80, 055202 (2009).

[78] E. F. McNicoll et al. [Crystal Ball at MAMI Collaboration], "Study of the $\gamma p \rightarrow \eta p$ Reaction with the Crystal Ball Detector at the Mainz Microtron (MAMI-C)," Phys. Rev. C 82, 035208 (2010), Erratum: [Phys. Rev. C 84, 029901 (2011)].

[79] B. Krusche et al., "New Threshold Photoproduction of $\eta$ Mesons off the Proton," Phys. Rev. Lett. 74, 3736 (1995).

[80] R. Bradford et al. [CLAS Collaboration], "Differential Cross Sections for $\gamma+p \rightarrow K^{+}+Y$ for $\Lambda$ and $\Sigma^{0}$ Hperons," Phys. Rev. C 73, 035202 (2006).

[81] A.V. Anisovich, V. Kleber, E. Klempt, V.A. Nikonov, A.V. Sarantsev and U. Thoma, "Baryon Resonances and Polarization Transfer in Hyperon Photoproduction," Eur. Phys. J. A 34, 243 (2007).

[82] Evgeny Golovach, Viktor Mokeev, and the CLAS Collaboration, "CLAS data on $\gamma p \rightarrow \pi^{+} \pi^{-} p$ ", private communication to BnGa.

[83] Volker Crede and the CLAS Collaboration, "CLAS polarization data on $\gamma p \rightarrow \pi^{+} \pi^{-} p$ ", private communication to $\mathrm{BnGa}$.

[84] R. Ewald et al. [CBELSA/TAPS Collaboration], "Measurement of Polarization Observables in $K_{s}^{0} \Sigma^{+}$ Photoproduction off the Proton," Phys. Lett. B 738, 268 (2014).

[85] E. Aker et al. [Crystal Barrel Collaboration], "The Crystal Barrel Spectrometer at LEAR," Nucl. Instrum. Meth. A 321, 69 (1992).
[86] A. Thiel et al., "Well-established Nucleon Resonances Revisited by Double-polarization Measurements," Phys. Rev. Lett. 109, 102001 (2012).

[87] A. Thiel et al. [CBELSA/TAPS Collaboration], "Double-polarization Observable G in Neutral-pion Photoproduction off the Proton," arXiv:1604.02922 [nucl-ex].

[88] A. Wilson et al. [CBELSA/TAPS Collaboration], "Photoproduction of $\omega$ mesons off the proton," Phys. Lett. B 749, 407 (2015).

[89] J. Junkersfeld et al. [CB-ELSA Collaboration], "Photoproduction of $\pi^{0} \omega$ off Protons for $E_{\gamma}<3 \mathrm{GeV}$," Eur. Phys. J. A 31, 365 (2007).

[90] D. Elsner et al., "Linearly Polarized Photon Beams at ELSA and Measurement of the Beam Asymmetry in $\pi^{0}$-photoproduction off the Proton," Eur. Phys. J. A 39, 373 (2009).

[91] N. Sparks et al. [CBELSA/TAPS Collaboration], "Measurement of the Beam Asymmetry $\Sigma$ in the Forward Direction for $\vec{\gamma} p \rightarrow p \pi^{0}$ Photoproduction", Phys. Rev. C 81, 065210 (2010).

[92] F. Klein et al. [CBELSA/TAPS Collaboration], "Beam Asymmetry in Near Threshold $\omega$ Photoproduction off the Proton," Phys. Rev. D 78, 117101 (2008).

[93] M. Hilt, B. C. Lehnhart, S. Scherer and L. Tiator, "Pion Photo- and Electroproduction in Relativistic Baryon Chiral Perturbation Theory and the Chiral MAID Interface," Phys. Rev. C 88, 055207 (2013).

[94] R. L. Workman, M. W. Paris, W. J. Briscoe and I. I. .Strakovsky, "Unified Chew-Mandelstam SAID Analysis of Pion Photoproduction Data," Phys. Rev. C 86, 015202 (2012).

[95] D. Rönchen et al., "Photocouplings at the Pole from Pion Photoproduction,” Eur. Phys. J. A 50, 101 (2014) Erratum: [Eur. Phys. J. A 51, 63 (2015)].

[96] O. Bartalini et al., "Measurement of $\pi^{0}$ Photoproduction on the Proton from $550 \mathrm{MeV}$ to $1500 \mathrm{MeV}$ at GRAAL," Eur. Phys. J. A 26, 399 (2005).

[97] R. L. Workman, private communication.

[98] D. Roenchen, private communication.

[99] A. V. Anisovich et al., "The Impact of New Polarization Data from Bonn, Mainz and Jefferson Laboratory on $\gamma p \rightarrow \pi N$ Multipoles," Eur. Phys. J. A 52, 284 (2016).

[100] M. Gottschall et al. [CBELSA/TAPS Collaboration], "First Measurement of the Helicity Asymmetry for $\gamma p \rightarrow p \pi^{0}$ in the Resonance Region," Phys. Rev. Lett. 112, 012003 (2014).

[101] M. Gottschall et al. [CBELSA/TAPS Collaboration], "Double Polarization Observable E for the Reaction $\gamma p \rightarrow \pi^{0} p$ ", in preparation.

[102] J. Hartmann et al., "The N(1520)3/2- Helicity Amplitudes from an Energy-independent Multipole Analysis Based on new Polarization Data on Photoproduction of Neutral Pions," Phys. Rev. Lett. 113, 062001 (2014).

[103] J. Hartmann et al. [CBELSA/TAPS Collaboration], "The Polarization Observables T, P, and $\mathrm{H}$ and their Impact on $\gamma p \rightarrow p \pi^{0}$ Multipoles," Phys. Lett. B 748, 
$212(2015)$.

[104] V.G. Gorbenko et al., Pisma Zh. Eksp. Teor. Fiz. 19, 659 (1974), V.G. Gorbenko et al., Yad. Fiz. 27, 1204 (1978), A.A. Belyaev et al., Nucl. Phys. B 213, 201 (1983), J.O. Maloy, Ph.D. Thesis, 1961. 141pp, V.G. Gorbenko et al., Pisma Zh. Eksp. Teor. Fiz. 22, 393 (1975), P.J. Bussey et al., Nucl. Phys. B 154, 492 (1979), S. Kato et al., Nucl. Phys. B 168, 1 (1980), A.S. Bratashevsky et al., Nucl. Phys. B 166, 525 (1980), A.S. Bratashevsky et al., Ukr. Fiz. Zh. (Russ.Ed.) 31, 1306 (1986)

[105] Y. Wunderlich, F. Afzal, A. Thiel, and R. Beck, "Determining the dominant partial wave contributions from angular distributions of single- and doublepolarization observables in pseudoscalar meson photoproduction," arXiv:1611.01031 [physics.data-an].

[106] PhD thesis Yannik Wunderlich, Bonn University, in prep.

[107] G.F. Chew, M.L. Goldberger, F.E. Low, Y. Nambu, "Relativistic Dispersion Relation Approach to Photomeson Production," Phys. Rev. 106, 1345 (1957).

[108] A. M. Sandorfi, S. Hoblit, H. Kamano and T.S. H. Lee, "Determining Pseudoscalar Meson Photoproduction Amplitudes from Complete Experiments," J. Phys. G 38, 053001 (2011).

[109] M. Abramowitz and I.A. Stegun, Handbook of Mathematical Functions, Dover Publishing (1972).

[110] J. Müller, J. Hartmann, M. Grüner et al. [CBELSA/TAPS Collaboration], "New Data on $\vec{\gamma} \vec{p} \rightarrow$ $\eta p$ with Polarized Photons and Protons and their Implications for $N^{*} \rightarrow N \eta$ Decays", subm. to Phys. Lett. B.

[111] PhD thesis Jonas Müller, Bonn University, in prep.

[112] PhD thesis Marcus Grüner, Bonn University, 2016

[113] PhD thesis Jan Hartmann, Bonn University, in prep.

[114] C. S. Akondi et al., "Measurement of the Transverse Target and Beam-target Asymmetries in $\eta \mathrm{Me}-$ son Photoproduction at MAMI,' Phys. Rev. Lett. 113, 102001 (2014).

[115] I. Senderovich et al. [CLAS Collaboration], "First Measurement of the Helicity Asymmetry E in $\eta$ Photoproduction on the Proton," Phys. Lett. B 775, 64 (2016).

[116] N. Kaiser, P. B. Siegel and W. Weise, "Chiral Dynamics and the S11 (1535) Nucleon Resonance," Phys. Lett. B 362, 23 (1995).

[117] M. Mai, P. C. Bruns and U.-G. Meißner, "Pion Photoproduction off the Proton in a Gauge-invariant Chiral Unitary Framework," Phys. Rev. D 86, 094033 (2012).

[118] B. S. Zou, "On the Nature of the Lowest $1 / 2^{-}$ Baryon Nonet and Decuplet," Eur. Phys. J. A 35, 325 (2008).

[119] E. Gutz et al. [CBELSA/TAPS Collaboration], "Measurement of the Beam Asymmetry $\Sigma$ in $\pi^{0} \eta$ Production off the Proton with the CBELSA/TAPS Experiment", Eur. Phys. J. A 35, 291 (2008).

[120] A. Fix, V. L. Kashevarov, A. Lee and M. Ostrick, "Isobar Model Analysis of $\pi^{0}-\eta$ Photoproduction on Protons,” Phys. Rev. C 82, 035207 (2010).
[121] A. Fix and H. Arenhövel, "Polarization Observables in $\pi^{0} \eta$-photoproduction on the Proton," Phys. Rev. C 83, 015503 (2011).

[122] M. Döring, E. Oset and U.-G. Meißner, "Evaluation of the Polarization Observables $I^{S}$ and $I^{C}$ in the Reaction $\gamma p \rightarrow \pi^{0} \eta p$," Eur. Phys. J. A 46, 315 (2010).

[123] H. Arenhovel, W. Leidemann, E. L. Tomusiak, "General Survey of Polarization Observables in Deuteron Electrodisintegration," Eur. Phys. J. A 23, 147 (2005).

[124] V. Kuznetsov et al., "Evidence for a Narrow Structure at $W \approx 1.68 \mathrm{GeV}$ in $\eta$ Photoproduction off the Neutron,” Phys. Lett. B 647, 23 (2007).

[125] I. Jaegle et al. [CBELSA/TAPS Collaboration], "Quasi-free Photoproduction of $\eta$-mesons of the Neutron”, Phys. Rev. Lett. 100, 252002 (2008).

[126] I. Jaegle et al. [CBELSA/TAPS Collaboration], "Photoproduction of $\eta^{\prime}$-mesons off the Deuteron", Eur. Phys. J. A 4711 (2011).

[127] I. Jaegle et al. [CBELSA/TAPS Collaboration], "Quasi-free Photoproduction of $\eta$-mesons off the Deuteron”, Eur. Phys. J. A 4789 (2011).

[128] D. Werthmüller et al. [A2 Collaboration], "Narrow Structure in the Excitation Function of $\eta$ Photoproduction off the Neutron," Phys. Rev. Lett. 111, 232001 (2013).

[129] D. Werthmüller et al. [A2 Collaboration], "Quasifree Photoproduction of $\eta$ Mesons off Protons and Neutrons," Phys. Rev. C 90, 015205 (2014).

[130] K. S. Choi, S. i. Nam, A. Hosaka and H. C. Kim, "A New $N^{*}(1675)$ Resonance in the $\gamma N \rightarrow \eta N$ reaction," Phys. Lett. B 636, 253 (2006).

[131] V. Shklyar, H. Lenske and U. Mosel, "Etaphotoproduction in the Resonance Energy Region," Phys. Lett. B 650, 172 (2007).

[132] R. Shyam and O. Scholten, "Photoproduction of $\eta$ Meson within a Coupled-channels K-matrix Approach,” Phys. Rev. C 78, 065201 (2008).

[133] A. V. Anisovich, I. Jaegle, E. Klempt, B. Krusche, V. A. Nikonov, A. V. Sarantsev and U. Thoma, "Photoproduction of $\eta$ mesons off Neutrons from a Deuteron Target,” Eur. Phys. J. A 41, 13 (2009).

[134] M. Doring and K. Nakayama, "On the Cross Section Ratio $\sigma(n) / \sigma(p)$ in $\eta$ Photoproduction," Phys. Lett. B 683, 145 (2010).

[135] A. V. Anisovich, E. Klempt, V. Kuznetsov, V. A. Nikonov, M. V. Polyakov, A. V. Sarantsev and U. Thoma, "Study of the Narrow Structure at $1685 \mathrm{MeV}$ in $\gamma p \rightarrow \eta p$," Phys. Lett. B 719, 89 (2013).

[136] A. V. Anisovich, E. Klempt, B. Krusche, V. A. Nikonov, A. V. Sarantsev, U. Thoma and D. Werthmüller, "Interference Phenomena in the $J^{P}=1 / 2^{-}$Wave in $\eta$ Photoproduction," Eur. Phys. J. A 51, 72 (2015).

[137] A.V. Anisovich, V. Burkert, N. Compton, K. Hicks, E. Klempt, V.A. Nikonov, A.V. Sarantsev, and U. Thoma, "Neutron helicity amplitudes", in preparation. 
[138] PhD thesis Philipp Mahlberg, Bonn University, in preparation.

[139] PhD thesis Tobias Seifen, Bonn University, in preparation.
[140] K. M. Watson, “The Hypothesis of Charge Independence for Nuclear Phenomena," Phys. Rev. 85, 852 (1952). 